\title{
SI: QM/MM Study on Mechanistic Photophysics of Alloxazine Chromophore in Aqueous Solution
}

\author{
Xue-Ping Chang, Xiao-Ying Xie, Shi-Yun Lin, and Ganglong Cui* \\ Key Laboratory of Theoretical and Computational Photochemistry, Ministry of Education, \\ College of Chemistry, Beijing Normal University, Beijing 100875, China \\ Email: ganglong.cui@bnu.edu.cn
}

\section{Table of Contents}

1. Active Orbitals in CASSCF and MS-CASPT2 Computations

2. Cartesian Coordinates of All Optimized Structures and Paths 


\title{
1. Active Orbitals in CASSCF and MS-CASPT2 Computations
}

\author{
$\operatorname{CASSCF}(14,10)$
}

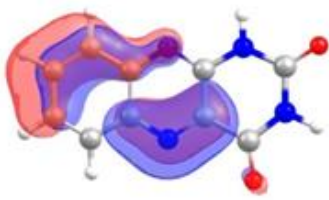

$\pi$

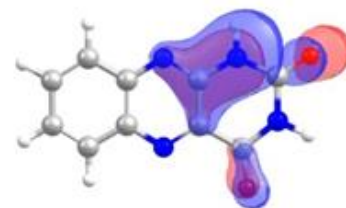

$\pi$

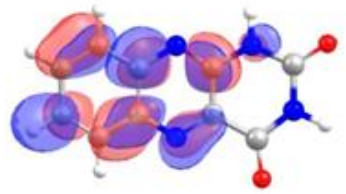

$\pi *$

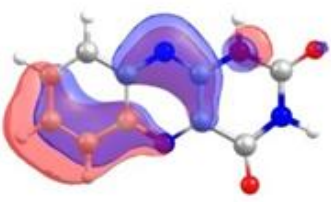

$\pi$

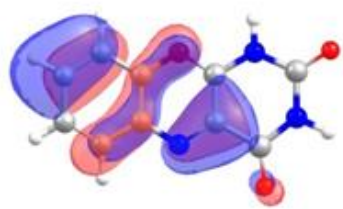

$\pi_{\mathrm{H}-1}$

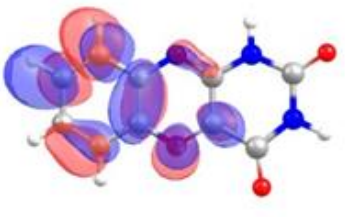

$\pi *$

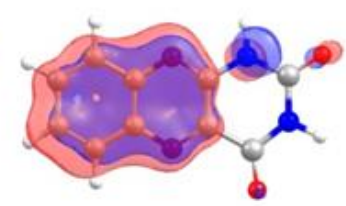

$\pi$

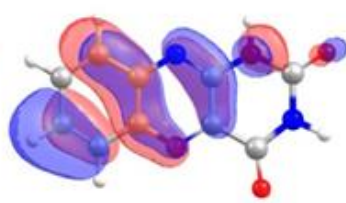

$\pi_{\mathrm{H}}$

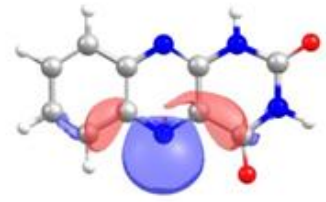

$\mathbf{n}_{\mathrm{N}}$

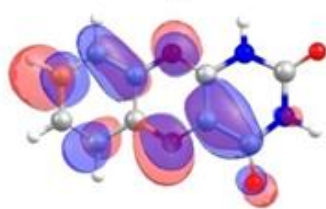

$\pi_{\mathrm{L}} *$

$\operatorname{CASSCF}(16,12)$

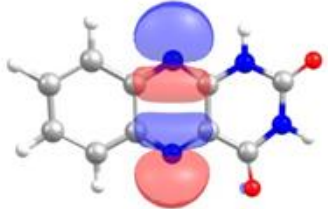

$\mathbf{n}_{\mathrm{N}-\mathrm{N}}$

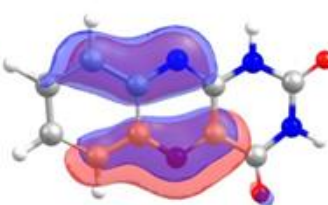

$\pi$

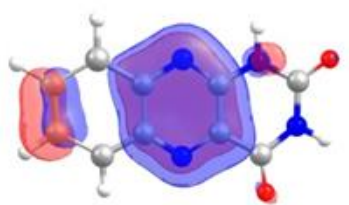

$\pi$

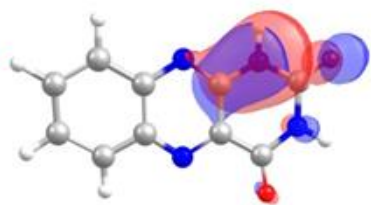

$\pi$

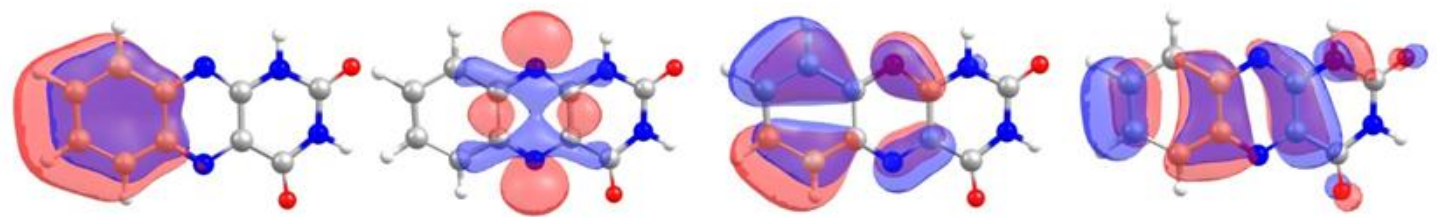

$\pi$

$\mathrm{n}_{\mathrm{N}+\mathrm{N}}$

$\pi_{\mathrm{H}-1}$

$\pi_{H}$

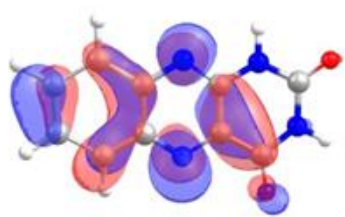

$\pi_{\mathrm{L}} *$

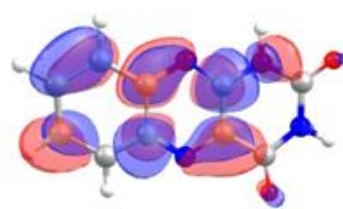

$\pi *$

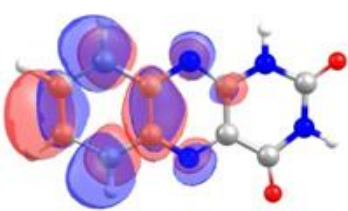

$\pi *$

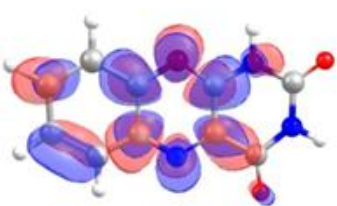

$\pi *$

Figure. S1: Active space used in the CASSCF geometry optimizations and MS-CASPT2 single-point energy computations. 
2. Cartesian Coordinates of All Optimized Structures and Paths (QM part) In xyz format (unit: angström)

CASSCF method

So

C

$-3.363964400$

$-1.750876370$

0.013440110

C

$-2.968129650$

$-0.472224470$

0.105136090

C

$-1.573486330$

$-0.155086600$

0.055278550

C

$-0.617454080$

$-1.199882300$

$-0.062302620$

C

$-1.069120020$

$-2.539635410$

$-0.169264010$

C

$-2.411715640$

$-2.801558750$

$-0.137175370$

C

1.056517480

0.303454450

$-0.004639850$

C

0.115322400

1.365712700

0.073615020

C

0.608109850

2.776369500

0.119082080

C

2.924683160

1.864345020

0.047135000

$\mathrm{H}$

$-3.666008950$

0.333374600

0.221015670

H

$-0.339973070$

$-3.319300700$

$-0.276849240$

$\mathrm{H}$

$-2.754044060$

$-3.814880890$

$-0.227360860$

$\mathrm{H}$

2.370001830

3.791039240

0.121890310

$\mathrm{H}$

$-4.409755140$

$-1.997944010$

0.048697960

N

0.701965410

$-0.931927730$

$-0.081623990$

$\mathrm{N}$

$-1.171102180$

1.135999190

0.105850290

$\mathrm{N}$

2.400962820

0.605852380

0.004416700 


$\begin{array}{lrrr}\mathrm{N} & 1.985368290 & 2.872406570 & 0.096269570 \\ \mathrm{O} & 4.094905410 & 2.086128280 & 0.044040730 \\ \mathrm{O} & -0.086938050 & 3.734905190 & 0.166483630 \\ \mathrm{H} & 3.051959200 & -0.148154070 & -0.046203890\end{array}$

${ }^{1} n \pi^{*}$

C

$-3.382538170$

$-1.810058950$

0.012263870

C

$-3.005032300$

$-0.499742040$

0.105002420

C

$-1.639744460$

$-0.178868010$

0.057974690

C

$-0.662116140$

$-1.198651880$

$-0.056713760$

C

$-1.099337790$

$-2.522311230$

$-0.157225400$

C

$-2.430880140$

$-2.831025260$

$-0.130022260$

C

1.068456030

0.320809660

$-0.004459930$

C

0.178992700

1.430068030

0.078317880

C

0.647146430

2.800828700

0.122310370

C

2.953964940

1.876068580

0.039621980

$\mathrm{H}$

$-3.730325050$

0.281713400

0.217489600

$\mathrm{H}$

$-0.351639330$

$-3.285838550$

$-0.260597400$

$\mathrm{H}$

$-2.743732640$

$-3.853181090$

$-0.218721230$

H

2.415824560

3.810238670

0.114470250

H

$-4.427954170$

$-2.058704220$

0.045159290

$\mathrm{N}$

0.700982310

$-0.918283760$

$-0.075749480$ 


\begin{tabular}{|c|c|c|c|}
\hline $\mathrm{N}$ & -1.120857060 & 1.064455110 & 0.104895450 \\
\hline $\mathrm{N}$ & 2.421612100 & 0.622197290 & -0.000183520 \\
\hline $\mathrm{N}$ & 2.026356220 & 2.894234510 & 0.091867410 \\
\hline 0 & 4.127587400 & 2.088157370 & 0.031981440 \\
\hline 0 & -0.057666870 & 3.762961630 & 0.174459350 \\
\hline $\mathrm{H}$ & 3.068025690 & -0.134820840 & -0.053147800 \\
\hline \multicolumn{4}{|c|}{${ }^{1} \pi \pi^{*}$} \\
\hline C & -3.366623380 & -1.821868800 & 0.012994170 \\
\hline C & -2.905464070 & -0.454858040 & 0.111923950 \\
\hline C & -1.556781490 & -0.110959460 & 0.054358470 \\
\hline C & -0.609258370 & -1.191146910 & -0.061409190 \\
\hline C & -1.073402830 & -2.531698390 & -0.171381120 \\
\hline C & -2.455319410 & -2.850571610 & -0.138876220 \\
\hline C & 1.050916380 & 0.317986760 & -0.001113780 \\
\hline C & 0.117652650 & 1.408875710 & 0.069252720 \\
\hline C & 0.605132280 & 2.775842770 & 0.110702930 \\
\hline C & 2.920340470 & 1.876943870 & 0.048095130 \\
\hline $\mathrm{H}$ & -3.616115150 & 0.338460570 & 0.235473590 \\
\hline $\mathrm{H}$ & -0.333903770 & -3.300317590 & -0.282962080 \\
\hline $\mathrm{H}$ & -2.772337170 & -3.869034620 & -0.235136140 \\
\hline 1 & 2.379552990 & 3.800976470 & 0.109476960 \\
\hline
\end{tabular}




$\begin{array}{llll}\mathrm{H} & -4.422675100 & -2.009573600 & 0.051173700 \\ \mathrm{~N} & 0.725504780 & -0.938228630 & -0.070390600 \\ \mathrm{~N} & -1.205818640 & 1.161336970 & 0.106934970 \\ \mathrm{~N} & 2.372155980 & 0.610219470 & 0.010945940 \\ \mathrm{~N} & 2.002861830 & 2.880174220 & 0.088321260 \\ \mathrm{O} & 4.099936760 & 2.048005600 & 0.044341240 \\ \mathrm{O} & -0.058437890 & 3.766581770 & 0.156496780 \\ \mathrm{H} & 3.017187170 & -0.150336090 & -0.033618930\end{array}$

$\begin{array}{llll}{ }^{3} \pi^{*} & & & \\ \text { C } & -3.3838444630 & -1.799788920 & 0.013881240 \\ \text { C } & -2.999875880 & -0.494598770 & 0.104504680 \\ \text { C } & -1.629952700 & -0.184259500 & 0.054892490 \\ \text { C } & -0.651738080 & -1.195397030 & -0.058617720 \\ \text { C } & -1.098092830 & -2.522445700 & -0.158757270 \\ \text { C } & -2.4284144490 & -2.822214440 & -0.129478770 \\ \text { C } & 1.064902510 & 0.315615710 & -0.005295760 \\ \text { C } & 0.178834760 & 1.423966000 & 0.077351610 \\ \text { C } & 0.639176880 & 2.793191340 & 0.125159740 \\ \text { C } & 2.946699360 & 1.875897940 & 0.044017800 \\ \text { H } & -3.719721660 & 0.292095710 & 0.217083420 \\ \text { H } & -0.354634410 & -3.289752320 & -0.262728630\end{array}$




\begin{tabular}{|c|c|c|c|}
\hline $\mathrm{H}$ & -2.744918990 & -3.843589670 & -0.217129750 \\
\hline $\mathrm{H}$ & 2.405498270 & 3.809142200 & 0.122699390 \\
\hline $\mathrm{H}$ & -4.428966410 & -2.047821310 & 0.048603080 \\
\hline N & 0.705352150 & -0.925197260 & -0.077683620 \\
\hline $\mathrm{N}$ & -1.123871740 & 1.072887050 & 0.100308840 \\
\hline N & 2.415714200 & 0.620766220 & 0.000863350 \\
\hline N & 2.018716610 & 2.892323870 & 0.097378910 \\
\hline 0 & 4.120741400 & 2.087803140 & 0.038199360 \\
\hline 0 & -0.068252520 & 3.755324430 & 0.178856270 \\
\hline $\mathrm{H}$ & 3.063305860 & -0.135173760 & -0.052311010 \\
\hline \multicolumn{4}{|c|}{$3 \pi \pi^{*}$} \\
\hline C & -3.385326320 & -1.860425350 & 0.002022550 \\
\hline C & -2.956808140 & -0.458518250 & 0.103733530 \\
\hline C & -1.586541110 & -0.111211550 & 0.048118740 \\
\hline C & -0.607383970 & -1.167278260 & -0.061269030 \\
\hline C & -1.077507480 & -2.524341900 & -0.165503730 \\
\hline C & -2.492414560 & -2.835865970 & -0.133765340 \\
\hline C & 1.028287530 & 0.384548430 & -0.001792400 \\
\hline C & 0.124395530 & 1.407398210 & 0.065829600 \\
\hline$C$ & 0.621633360 & 2.798783580 & 0.114799130 \\
\hline C & 2.934811670 & 1.867953280 & 0.045685910 \\
\hline
\end{tabular}




$\begin{array}{lrrr}\mathrm{H} & -3.681238070 & 0.320710570 & 0.220697030 \\ \mathrm{H} & -0.341470680 & -3.295238630 & -0.273938170 \\ \mathrm{H} & -2.787371090 & -3.862956270 & -0.224224000 \\ \mathrm{H} & 2.396454240 & 3.798141720 & 0.121364660 \\ \mathrm{H} & -4.438367040 & -2.070940360 & 0.031498260 \\ \mathrm{~N} & 0.661609670 & -0.932977870 & -0.078802840 \\ \mathrm{~N} & -1.209500540 & 1.173905510 & 0.092951370 \\ \mathrm{~N} & 2.385647530 & 0.622525390 & 0.002122110 \\ \mathrm{~N} & 2.006810470 & 2.882022180 & 0.095033030 \\ \mathrm{O} & 4.111449850 & 2.071104970 & 0.042835990 \\ \mathrm{O} & -0.053319920 & 3.776291090 & 0.161856790 \\ \mathrm{H} & 3.010873520 & -0.151451140 & -0.047476690\end{array}$

\begin{tabular}{lrrr}
\multicolumn{1}{l}{${ }^{*} \pi \pi^{*} / 3 \pi \pi^{*}$} & & \\
C & -3.369040030 & -1.820073360 & 0.014039230 \\
C & -2.914585150 & -0.457042220 & 0.111906010 \\
C & -1.565607330 & -0.115567850 & 0.054066810 \\
C & -0.614152230 & -1.192935260 & -0.061597530 \\
C & -1.076109570 & -2.529049600 & -0.170236160 \\
C & -2.455181540 & -2.846823410 & -0.137483220 \\
C & 1.051254020 & 0.317674920 & -0.001893630 \\
C & 0.121446140 & 1.410146810 & 0.068585460
\end{tabular}




$\begin{array}{lrrr}\mathrm{C} & 0.608096360 & 2.777322540 & 0.111366600 \\ \mathrm{C} & 2.922396450 & 1.876824990 & 0.047495970 \\ \mathrm{H} & -3.627039160 & 0.334832460 & 0.234459510 \\ \mathrm{H} & -0.336632770 & -3.298001240 & -0.280971980 \\ \mathrm{H} & -2.771409030 & -3.865827050 & -0.233306120 \\ \mathrm{H} & 2.381681690 & 3.801663380 & 0.110344570 \\ \mathrm{H} & -4.424493640 & -2.011989030 & 0.052377910 \\ \mathrm{~N} & 0.722480840 & -0.936955120 & -0.070913980 \\ \mathrm{~N} & -1.200892140 & 1.1558336880 & 0.105976850 \\ \mathrm{~N} & 2.375086590 & 0.611274220 & 0.009933000 \\ \mathrm{~N} & 2.004308780 & 2.881084180 & 0.088693380 \\ \mathrm{O} & 4.101631320 & 2.050516150 & 0.043514620 \\ \mathrm{O} & -0.059042990 & 3.765569540 & 0.158224990 \\ \mathrm{H} & 3.020176220 & -0.148988240 & -0.034909660\end{array}$

${ }^{1} \pi \pi^{*} / 1 n \pi^{*}$

$\begin{array}{llll}\text { C } & -3.370881960 & -1.811221230 & 0.012313900 \\ \text { C } & -2.945297800 & -0.474588290 & 0.105394580 \\ \text { C } & -1.609536230 & -0.146951640 & 0.052993200 \\ \text { C } & -0.626389170 & -1.198478820 & -0.063119830 \\ \text { C } & -1.081568660 & -2.534209250 & -0.168104600 \\ \text { C } & -2.442901200 & -2.843231330 & -0.135769830\end{array}$




$\begin{array}{lrrr}\mathrm{C} & 1.046938300 & 0.329182350 & -0.001737060 \\ \mathrm{C} & 0.143424760 & 1.417565490 & 0.074085890 \\ \mathrm{C} & 0.620614500 & 2.784209120 & 0.119895900 \\ \mathrm{C} & 2.928935820 & 1.874607510 & 0.047197530 \\ \mathrm{H} & -3.663530450 & 0.313270080 & 0.221609320 \\ \mathrm{H} & -0.339055870 & -3.300759050 & -0.277875900 \\ \mathrm{H} & -2.765413990 & -3.860877170 & -0.226386510 \\ \mathrm{H} & 2.393666400 & 3.803039230 & 0.122033380 \\ \mathrm{H} & -4.422381810 & -2.026638500 & 0.050462130 \\ \mathrm{~N} & 0.701877190 & -0.923399620 & -0.075049130 \\ \mathrm{~N} & -1.177982730 & 1.123650000 & 0.100759700 \\ \mathrm{~N} & 2.380840420 & 0.615443560 & 0.004387500 \\ \mathrm{~N} & 2.009789820 & 2.885111160 & 0.096656610 \\ \mathrm{O} & 4.107081190 & 2.057829520 & 0.041939030 \\ \mathrm{O} & -0.064173080 & 3.761565350 & 0.169723440 \\ \mathrm{H} & 3.021193970 & -0.147953310 & -0.047965760\end{array}$

\begin{tabular}{llll}
\multicolumn{2}{l}{${ }^{1} \mathrm{n} \pi^{*} / 3 \pi^{*}$} \\
C & -3.383927810 & -1.810763390 & 0.010646540 \\
C & -3.009420600 & -0.501518350 & 0.103495080 \\
C & -1.646749790 & -0.184443000 & 0.056800400 \\
C & -0.664074280 & -1.196817280 & -0.057890440
\end{tabular}




\begin{tabular}{|c|c|c|c|}
\hline C & -1.106086260 & -2.526771100 & -0.159165980 \\
\hline C & -2.429489690 & -2.833966410 & -0.132071420 \\
\hline C & 1.066971780 & 0.318462220 & -0.004414390 \\
\hline C & 0.182241400 & 1.437696220 & 0.079505920 \\
\hline C & 0.648591220 & 2.805395910 & 0.124719510 \\
\hline C & 2.953107500 & 1.877572370 & 0.041886850 \\
\hline $\mathrm{H}$ & -3.735260820 & 0.279419900 & 0.215541620 \\
\hline $\mathrm{H}$ & -0.356048100 & -3.288077110 & -0.262734360 \\
\hline $\mathrm{H}$ & -2.743500220 & -3.855810580 & -0.220649530 \\
\hline $\mathrm{H}$ & 2.418185470 & 3.813025050 & 0.118373020 \\
\hline $\mathrm{H}$ & -4.429209590 & -2.059563140 & 0.043637210 \\
\hline $\mathrm{N}$ & 0.698146670 & -0.916397880 & -0.076339580 \\
\hline $\mathrm{N}$ & -1.104492660 & 1.053779610 & 0.104163750 \\
\hline $\mathrm{N}$ & 2.419324130 & 0.623782310 & 0.000611940 \\
\hline $\mathrm{N}$ & 2.027707240 & 2.897599610 & 0.094846160 \\
\hline 0 & 4.126940340 & 2.087344300 & 0.034883500 \\
\hline 0 & -0.058324590 & 3.766675050 & 0.177658130 \\
\hline $\mathrm{H}$ & 3.065354250 & -0.133632790 & -0.052805800 \\
\hline
\end{tabular}

${ }^{1} \pi \pi^{*} / 3 n \pi^{*}$

$\begin{array}{llll}\text { C } & -3.366790830 & -1.820078540 & 0.013790010 \\ \text { C } & -2.907331060 & -0.454593280 & 0.112505050\end{array}$




\begin{tabular}{lrrr} 
C & -1.558258130 & -0.110437810 & 0.054451510 \\
C & -0.610124420 & -1.191343220 & -0.061380630 \\
C & -1.073674960 & -2.530853290 & -0.171132790 \\
C & -2.454822150 & -2.849239390 & -0.138409010 \\
C & 1.050692710 & 0.317462930 & -0.001298140 \\
C & 0.117416320 & 1.408849120 & 0.068818980 \\
C & 0.605465890 & 2.775851950 & 0.110445470 \\
C & 2.920321430 & 1.876241270 & 0.047775850 \\
H & -3.618361560 & 0.338322520 & 0.236202950 \\
H & -0.334157420 & -3.299521830 & -0.282634240 \\
H & -2.772119980 & -3.867675860 & -0.234731300 \\
H & 2.380263610 & 3.800437360 & 0.109114950 \\
$\mathrm{H}$ & -4.422778530 & -2.008427020 & 0.052221380 \\
$\mathrm{~N}$ & 0.725147420 & -0.938650730 & -0.070361380 \\
$\mathrm{~N}$ & -1.206674750 & 1.162026510 & 0.106991470 \\
$\mathrm{~N}$ & 2.371976740 & 0.609649020 & 0.010756630 \\
$\mathrm{~N}$ & 2.003061300 & 2.879803100 & 0.088006150 \\
$\mathrm{O}$ & 4.099874310 & 2.047162600 & 0.043945650 \\
$\mathrm{H}$ & -0.058146290 & 3.766436470 & 0.156376220 \\
\hline & 3.0169966130 & -0.150973460 & -0.033590070
\end{tabular}




\begin{tabular}{|c|c|c|c|}
\hline C & -3.386402180 & -1.810200170 & 0.024235530 \\
\hline C & -2.999092280 & -0.495025730 & 0.108254850 \\
\hline C & -1.638476520 & -0.173476640 & 0.058423610 \\
\hline C & -0.661351640 & -1.203676930 & -0.055755580 \\
\hline C & -1.091746590 & -2.508471050 & -0.150690760 \\
\hline C & -2.442175370 & -2.820167030 & -0.119039150 \\
\hline C & 1.060976400 & 0.315767300 & -0.006356610 \\
\hline C & 0.165084590 & 1.415760430 & 0.074531330 \\
\hline C & 0.636239140 & 2.790679430 & 0.120058770 \\
\hline C & 2.944609820 & 1.872726690 & 0.041159630 \\
\hline $\mathrm{H}$ & -3.722588630 & 0.288733340 & 0.214661950 \\
\hline $\mathrm{H}$ & -0.350588390 & -3.278744740 & -0.250914210 \\
\hline $\mathrm{H}$ & -2.748374910 & -3.844219390 & -0.205661750 \\
\hline $\mathrm{H}$ & 2.401319530 & 3.806085510 & 0.115301570 \\
\hline $\mathrm{H}$ & -4.432415270 & -2.054467790 & 0.064305620 \\
\hline $\mathrm{N}$ & 0.703923110 & -0.926913780 & -0.074958690 \\
\hline $\mathrm{N}$ & -1.158362380 & 1.091079890 & 0.095662810 \\
\hline $\mathrm{N}$ & 2.413964080 & 0.618837160 & -0.000500400 \\
\hline $\mathrm{N}$ & 2.015020910 & 2.888514140 & 0.092672740 \\
\hline 0 & 4.117974990 & 2.086812740 & 0.035930930 \\
\hline 0 & -0.068669660 & 3.751950780 & 0.169972410 \\
\hline $\mathrm{H}$ & 3.060894240 & -0.138063030 & -0.050152960 \\
\hline
\end{tabular}




$\begin{array}{lrrr}{ }^{3} \pi \pi^{*} / g s & & \\ \text { C } & -3.497626120 & -1.576525330 & -0.486896350 \\ \text { C } & -3.054566600 & -0.432480060 & 0.366632400 \\ \text { C } & -1.658437150 & 0.021500850 & 0.106869420 \\ \text { C } & -0.735107160 & -1.072329920 & 0.100919030 \\ \mathrm{C} & -1.296176470 & -2.403625070 & -0.004391450 \\ \mathrm{C} & -2.643461700 & -2.590340950 & -0.536925530 \\ \mathrm{C} & 0.952003260 & 0.392705440 & 0.103622660 \\ \mathrm{C} & 0.073012420 & 1.454530040 & 0.023025500 \\ \mathrm{C} & 0.600925190 & 2.834646080 & -0.000504140 \\ \mathrm{C} & 2.888437720 & 1.853051450 & 0.090093490 \\ \mathrm{H} & -3.231530410 & -0.607520530 & 1.417977690 \\ \mathrm{H} & -0.628068940 & -3.236937450 & 0.092458200 \\ \mathrm{H} & -2.866950980 & -3.518792240 & -1.028541310 \\ \mathrm{H} & 2.394610880 & 3.796139730 & -0.028087640 \\ \mathrm{H} & -4.447975690 & -1.573850320 & -0.987696760 \\ \mathrm{~N} & 0.553618100 & -0.881801470 & 0.143751910 \\ \mathrm{~N} & -1.268722360 & 1.257033780 & 0.048324340 \\ \mathrm{~N} & 2.312912650 & 0.618666950 & 0.142644950 \\ \mathrm{~N} & 1.985620900 & 2.888620070 & 0.014386330 \\ & 4.068456490 & 2.029597970 & 0.110245600\end{array}$



0
$-0.056978500$
3.823681670
$-0.032664470$
H $\quad 2.925675910$
$-0.164238700$
0.211708850

LIIC path connecting the ${ }^{1} \pi \pi^{*}$ and the ${ }^{1} \pi \pi^{*} / 3 \pi \pi^{*}$ minima of alloxazine (10 points)

\section{Coord.1}

$\begin{array}{llll}\text { C } & -3.366623240 & -1.821869129 & 0.012994000 \\ \text { C } & -2.905464207 & -0.454858035 & 0.111924010 \\ \text { C } & -1.556781114 & -0.110959007 & 0.054358002 \\ \text { C } & -0.609258044 & -1.191147084 & -0.061409004 \\ \text { C } & -1.073403077 & -2.531698182 & -0.171381011 \\ \text { C } & -2.455319178 & -2.850572206 & -0.138876012 \\ \text { C } & 1.050916076 & 0.317987022 & -0.001113997 \\ \text { C } & 0.117653010 & 1.408876100 & 0.069253003 \\ \text { C } & 0.605132044 & 2.775843197 & 0.110703007 \\ \text { C } & 2.920340210 & 1.876944134 & 0.048095005 \\ \text { H } & -3.616115258 & 0.338461022 & 0.235474017 \\ \text { H } & -0.333904023 & -3.300318235 & -0.282962018 \\ \text { H } & -2.772337197 & -3.869035280 & -0.235136016 \\ \text { H } & 2.379553172 & 3.800976276 & 0.109477009 \\ \text { H } & -4.422675319 & -2.009574146 & 0.051174001\end{array}$




$\begin{array}{llll}\mathrm{N} & 0.725505051 & -0.938229066 & -0.070391004 \\ \mathrm{~N} & -1.205819089 & 1.161337084 & 0.106935006 \\ \mathrm{~N} & 2.372156168 & 0.610219046 & 0.010946000 \\ \mathrm{~N} & 2.002862147 & 2.880174207 & 0.088321006 \\ \mathrm{O} & 4.099937296 & 2.048006147 & 0.044341005 \\ \mathrm{O} & -0.058438007 & 3.766582273 & 0.156497012 \\ \mathrm{H} & 3.017187219 & -0.150336013 & -0.033619001\end{array}$

\section{Coord.2}

$\begin{array}{llll}\text { C } & -3.366989240 & -1.821873129 & 0.013047003 \\ \text { C } & -2.906563207 & -0.455241032 & 0.111882009 \\ \text { C } & -1.557832113 & -0.111487010 & 0.054333004 \\ \text { C } & -0.609751042 & -1.191227085 & -0.061385005 \\ \text { C } & -1.073741078 & -2.531303183 & -0.171229010 \\ \text { C } & -2.455344176 & -2.850253207 & -0.138762012 \\ \text { C } & 1.051017074 & 0.317974020 & -0.001157999 \\ \text { C } & 0.118137011 & 1.409057099 & 0.069193005 \\ \text { C } & 0.605552041 & 2.776015201 & 0.110722010 \\ \text { C } & 2.920688213 & 1.877020135 & 0.048052004 \\ \text { H } & -3.617461262 & 0.337877022 & 0.235315015 \\ \text { H } & -0.334121023 & -3.299855237 & -0.282709019 \\ \text { H } & -2.772126198 & -3.868821276 & -0.235017014\end{array}$




$\begin{array}{lrrr}\mathrm{H} & 2.379876171 & 3.801117275 & 0.109510009 \\ \mathrm{H} & -4.422989317 & -2.010005145 & 0.051219002 \\ \mathrm{~N} & 0.725255052 & -0.938122067 & -0.070391004 \\ \mathrm{~N} & -1.205277084 & 1.160736082 & 0.106815010 \\ \mathrm{~N} & 2.372494170 & 0.610421044 & 0.010893003 \\ \mathrm{~N} & 2.003158142 & 2.880318206 & 0.088328007 \\ \mathrm{O} & 4.100265296 & 2.048269148 & 0.044282002 \\ \mathrm{O} & -0.058425005 & 3.766461272 & 0.156576012 \\ \mathrm{H} & 3.017496216 & -0.150129009 & -0.033680005\end{array}$

\section{Coord.3}

$\begin{array}{llll}\text { C } & -3.367354240 & -1.821876130 & 0.013099000 \\ \text { C } & -2.907661208 & -0.455623035 & 0.111841008 \\ \text { C } & -1.5588883112 & -0.112014007 & 0.054308006 \\ \text { C } & -0.610244044 & -1.191307086 & -0.061361002 \\ \text { C } & -1.074078079 & -2.530908184 & -0.171077014 \\ \text { C } & -2.455369174 & -2.849933203 & -0.138649011 \\ \text { C } & 1.051118073 & 0.317962024 & -0.001203000 \\ \text { C } & 0.118621007 & 1.409239099 & 0.069134007 \\ \text { C } & 0.605971044 & 2.776185199 & 0.110742008 \\ \text { C } & 2.921035210 & 1.877096135 & 0.048008003 \\ \text { H } & -3.618806262 & 0.337294022 & 0.235156019\end{array}$




$\begin{array}{llll}\mathrm{H} & -0.334338022 & -3.299391239 & -0.282456019 \\ \mathrm{H} & -2.771914199 & -3.868606276 & -0.234897018 \\ \mathrm{H} & 2.380198170 & 3.801257274 & 0.109543008 \\ \mathrm{H} & -4.423301320 & -2.010436144 & 0.051263004 \\ \mathrm{~N} & 0.725005053 & -0.938014067 & -0.070391004 \\ \mathrm{~N} & -1.204735085 & 1.160135085 & 0.106694008 \\ \mathrm{~N} & 2.372830171 & 0.610622046 & 0.010840000 \\ \mathrm{~N} & 2.003454142 & 2.880460206 & 0.088334008 \\ \mathrm{O} & 4.100591296 & 2.048531149 & 0.044222004 \\ \mathrm{O} & -0.058412003 & 3.766339270 & 0.156654013 \\ \mathrm{H} & 3.017804218 & -0.149922011 & -0.033742003\end{array}$

\section{Coord. 4}

$\begin{array}{llll}\text { C } & -3.367718240 & -1.821879130 & 0.013152002 \\ \text { C } & -2.908759208 & -0.456006032 & 0.111799008 \\ \text { C } & -1.559934111 & -0.112542009 & 0.054284002 \\ \text { C } & -0.610736042 & -1.191387087 & -0.061338004 \\ \text { C } & -1.074415075 & -2.530512179 & -0.170925013 \\ \text { C } & -2.455394178 & -2.849613205 & -0.138535011 \\ \text { C } & 1.051219077 & 0.317949022 & -0.001247001 \\ \text { C } & 0.119105009 & 1.409420099 & 0.069074004 \\ \text { C } & 0.606390041 & 2.776356198 & 0.110761010\end{array}$




$\begin{array}{lrrr}\mathrm{C} & 2.921382213 & 1.877172136 & 0.047965002 \\ \mathrm{H} & -3.620152261 & 0.336710022 & 0.234997017 \\ \mathrm{H} & -0.334556022 & -3.298928235 & -0.282202019 \\ \mathrm{H} & -2.771702200 & -3.868392277 & -0.234778017 \\ \mathrm{H} & 2.380520169 & 3.801397273 & 0.109576008 \\ \mathrm{H} & -4.423614318 & -2.010867143 & 0.051308005 \\ \mathrm{~N} & 0.724754053 & -0.937907067 & -0.070391004 \\ \mathrm{~N} & -1.204193086 & 1.159534082 & 0.106574007 \\ \mathrm{~N} & 2.373167172 & 0.610823044 & 0.010786003 \\ \mathrm{~N} & 2.003749143 & 2.880603205 & 0.088341009 \\ \mathrm{O} & 4.100918295 & 2.048793150 & 0.044163001 \\ \mathrm{O} & -0.058399006 & 3.766218273 & 0.156733009 \\ \mathrm{H} & 3.018112215 & -0.149715012 & -0.033803001\end{array}$

\section{Coord.5}

$\begin{array}{llll}\text { C } & -3.368083240 & -1.821883131 & 0.013203999 \\ \text { C } & -2.909857209 & -0.456388035 & 0.111758007 \\ \text { C } & -1.560984110 & -0.113069006 & 0.054259004 \\ \text { C } & -0.611229044 & -1.191467088 & -0.061314006 \\ \text { C } & -1.074752076 & -2.530117180 & -0.170773013 \\ \text { C } & -2.455418176 & -2.849293206 & -0.138421010 \\ \text { C } & 1.051319076 & 0.317936025 & -0.001292002\end{array}$




$\begin{array}{lrrr}\mathrm{C} & 0.119589010 & 1.409601099 & 0.069014006 \\ \mathrm{C} & 0.606810044 & 2.776527201 & 0.110780008 \\ \mathrm{C} & 2.921729210 & 1.877248136 & 0.047921006 \\ \mathrm{H} & -3.621497260 & 0.336126022 & 0.234838015 \\ \mathrm{H} & -0.334773022 & -3.298464236 & -0.281949020 \\ \mathrm{H} & -2.771490201 & -3.868177278 & -0.234658015 \\ \mathrm{H} & 2.380843174 & 3.801536272 & 0.109610008 \\ \mathrm{H} & -4.423926321 & -2.011298142 & 0.051353006 \\ \mathrm{~N} & 0.724504054 & -0.937800068 & -0.070391004 \\ \mathrm{~N} & -1.203651087 & 1.158933085 & 0.106453010 \\ \mathrm{~N} & 2.373504173 & 0.611025046 & 0.010733001 \\ \mathrm{~N} & 2.004045144 & 2.880746205 & 0.088348004 \\ \mathrm{O} & 4.101245295 & 2.049055145 & 0.044104003 \\ \mathrm{O} & -0.058386004 & 3.766096272 & 0.156812010 \\ \mathrm{H} & 3.018421218 & -0.149508009 & -0.033864005\end{array}$

\section{Coord. 6}

$\begin{array}{llll}\text { C } & -3.368448240 & -1.821886131 & 0.013257001 \\ \text { C } & -2.910956209 & -0.456771032 & 0.111716006 \\ \text { C } & -1.562035114 & -0.113597009 & 0.054234006 \\ \text { C } & -0.611722042 & -1.191547083 & -0.061290002 \\ \text { C } & -1.075090078 & -2.529721181 & -0.170621012\end{array}$




$\begin{array}{lrrr}\mathrm{C} & -2.455443174 & -2.848973207 & -0.138307009 \\ \mathrm{C} & 1.051420075 & 0.317923024 & -0.001335998 \\ \mathrm{C} & 0.120073006 & 1.409782104 & 0.068955002 \\ \mathrm{C} & 0.607229041 & 2.776698199 & 0.110799005 \\ \mathrm{C} & 2.922076213 & 1.877324137 & 0.047878005 \\ \mathrm{H} & -3.622842259 & 0.335542022 & 0.234679018 \\ \mathrm{H} & -0.334990022 & -3.298000238 & -0.281696020 \\ \mathrm{H} & -2.771279197 & -3.867962278 & -0.234539019 \\ \mathrm{H} & 2.381165173 & 3.801676271 & 0.109643007 \\ \mathrm{H} & -4.424239319 & -2.011728146 & 0.051398002 \\ \mathrm{~N} & 0.724253050 & -0.937692068 & -0.070392004 \\ \mathrm{~N} & -1.203108087 & 1.158332083 & 0.106333009 \\ \mathrm{~N} & 2.373841169 & 0.611226044 & 0.010680003 \\ \mathrm{~N} & 2.004341144 & 2.880889210 & 0.088355005 \\ \mathrm{O} & 4.101571295 & 2.049317146 & 0.044045005 \\ \mathrm{O} & -0.058373003 & 3.765974270 & 0.156890010 \\ \mathrm{H} & 3.018729215 & -0.149301011 & -0.033926003\end{array}$

\section{Coord. 7}

$\begin{array}{llll}\text { C } & -3.368813240 & -1.821889131 & 0.013309998 \\ \text { C } & -2.912054210 & -0.457153034 & 0.111674011 \\ \text { C } & -1.563086113 & -0.114124006 & 0.054209002\end{array}$




\begin{tabular}{|c|c|c|c|}
\hline C & -0.612214044 & -1.191626084 & -0.061267004 \\
\hline C & -1.075427079 & -2.529326182 & -0.170469011 \\
\hline C & -2.455468178 & -2.848653203 & -0.138194009 \\
\hline C & 1.051521073 & 0.317911022 & -0.001380999 \\
\hline C & 0.120557007 & 1.409963104 & 0.068895004 \\
\hline C & 0.607649044 & 2.776869198 & 0.110819008 \\
\hline C & 2.922423210 & 1.877400137 & 0.047835004 \\
\hline $\mathrm{H}$ & -3.624188259 & 0.334959023 & 0.234520016 \\
\hline $\mathrm{H}$ & -0.335207027 & -3.297536239 & -0.281442020 \\
\hline $\mathrm{H}$ & -2.771067198 & -3.867747279 & -0.234419018 \\
\hline $\mathrm{H}$ & 2.381487172 & 3.801816276 & 0.109676007 \\
\hline $\mathrm{H}$ & -4.424551316 & -2.012159145 & 0.051442003 \\
\hline $\mathrm{N}$ & 0.724003050 & -0.937585068 & -0.070392004 \\
\hline $\mathrm{N}$ & -1.202566088 & 1.157731085 & 0.106212007 \\
\hline $\mathrm{N}$ & 2.374178170 & 0.611427047 & 0.010627001 \\
\hline $\mathrm{N}$ & 2.004636145 & 2.881032209 & 0.088361006 \\
\hline 0 & 4.101898295 & 2.049580147 & 0.043985001 \\
\hline 0 & -0.058360006 & 3.765852273 & 0.156969011 \\
\hline 11 & 3.019037217 & -0.149094012 & -0.033987001 \\
\hline
\end{tabular}

\section{Coord. 8}

$\begin{array}{llll}\text { C } & -3.369178240 & -1.821893132 & 0.013362001\end{array}$ 


\begin{tabular}{|c|c|c|c|}
\hline C & -2.913152210 & -0.457536032 & 0.111633010 \\
\hline C & -1.564136112 & -0.114652008 & 0.054184004 \\
\hline C & -0.612707042 & -1.191706085 & -0.061243006 \\
\hline C & -1.075764075 & -2.528930183 & -0.170317010 \\
\hline C & -2.455493176 & -2.848333204 & -0.138080008 \\
\hline C & 1.051621077 & 0.317898025 & -0.001425000 \\
\hline C & 0.121041009 & 1.410144103 & 0.068835006 \\
\hline C & 0.608068046 & 2.777039201 & 0.110838006 \\
\hline C & 2.922769213 & 1.877476138 & 0.047791003 \\
\hline $\mathrm{H}$ & -3.625533263 & 0.334375023 & 0.234361015 \\
\hline $\mathrm{H}$ & -0.335424026 & -3.297072236 & -0.281189021 \\
\hline $\mathrm{H}$ & -2.770855199 & -3.867532279 & -0.234300016 \\
\hline $\mathrm{H}$ & 2.381810171 & 3.801956275 & 0.109709006 \\
\hline $\mathrm{H}$ & -4.424864319 & -2.012590144 & 0.051487004 \\
\hline $\mathrm{N}$ & 0.723753051 & -0.937478069 & -0.070392004 \\
\hline $\mathrm{N}$ & -1.202024089 & 1.157130083 & 0.106092006 \\
\hline $\mathrm{N}$ & 2.374515172 & 0.611629044 & 0.010572998 \\
\hline $\mathrm{N}$ & 2.004932145 & 2.881175209 & 0.088368007 \\
\hline 0 & 4.102225294 & 2.049842148 & 0.043926003 \\
\hline 0 & -0.058347004 & 3.765730271 & 0.157048012 \\
\hline $\mathrm{H}$ & 3.019345220 & -0.148888009 & -0.034048005 \\
\hline
\end{tabular}




\section{Coord.9}

$\begin{array}{llll}\text { C } & -3.369542245 & -1.821896132 & 0.013415003 \\ \text { C } & -2.914250210 & -0.457918034 & 0.111591009 \\ \text { C } & -1.565187111 & -0.115179011 & 0.054160006 \\ \text { C } & -0.613200044 & -1.191786086 & -0.061219002 \\ \text { C } & -1.076101076 & -2.528535184 & -0.170164014 \\ \text { C } & -2.455517174 & -2.848013205 & -0.137966008 \\ \text { C } & 1.051722076 & 0.317885023 & -0.001470001 \\ \text { C } & 0.121525010 & 1.410325103 & 0.068776003 \\ \text { C } & 0.608487044 & 2.777210200 & 0.110857009 \\ \text { C } & 2.923116210 & 1.877552133 & 0.047748002 \\ \text { H } & -3.626878262 & 0.333791023 & 0.234202018 \\ \text { H } & -0.335641026 & -3.296608237 & -0.280936021 \\ \text { H } & -2.770643200 & -3.867318280 & -0.234180015 \\ \text { H } & 2.382132170 & 3.802096274 & 0.109742006 \\ \text { H } & -4.425176317 & -2.013021143 & 0.051532005 \\ \mathrm{~N} & 0.723502052 & -0.937370069 & -0.070392004 \\ \mathrm{~N} & -1.201482085 & 1.156529086 & 0.105971009 \\ \mathrm{~N} & 2.374851173 & 0.611830041 & 0.010520001 \\ \mathrm{~N} & 2.005228146 & 2.881317208 & 0.0883740008 \\ \mathrm{O} & 4.102551294 & 2.050104149 & 0.043867005 \\ \mathrm{O} & -0.058334002 & 3.765608270 & 0.157127013\end{array}$




\section{Coord.10}

\begin{tabular}{|c|c|c|c|}
\hline C & -3.369915241 & -1.821898133 & 0.013469000 \\
\hline C & -2.915356212 & -0.458300031 & 0.111551009 \\
\hline C & -1.566246111 & -0.115706008 & 0.054136002 \\
\hline C & -0.613701043 & -1.191865087 & -0.061194004 \\
\hline C & -1.076447079 & -2.528138185 & -0.170011013 \\
\hline C & -2.455550179 & -2.847691206 & -0.137851012 \\
\hline C & 1.051814074 & 0.317873022 & -0.001513002 \\
\hline C & 0.122000010 & 1.410506103 & 0.068717005 \\
\hline C & 0.608898045 & 2.777381198 & 0.110877006 \\
\hline C & 2.923454212 & 1.877628133 & 0.047705001 \\
\hline $\mathrm{H}$ & -3.628231263 & 0.333208023 & 0.234044016 \\
\hline $\mathrm{H}$ & -0.335867022 & -3.296143239 & -0.280681021 \\
\hline $\mathrm{H}$ & -2.770439197 & -3.867101280 & -0.234059018 \\
\hline $\mathrm{H}$ & 2.382445173 & 3.802235273 & 0.109776011 \\
\hline $\mathrm{H}$ & -4.425496321 & -2.013450147 & 0.051578002 \\
\hline$N$ & 0.723243051 & -0.937262069 & -0.070391004 \\
\hline $\mathrm{N}$ & -1.200948087 & 1.155928083 & 0.105852008 \\
\hline $\mathrm{N}$ & 2.375179173 & 0.612032044 & 0.010467999 \\
\hline $\mathrm{N}$ & 2.005514145 & 2.881460208 & 0.088382004 \\
\hline
\end{tabular}



0
4.102868298
2.050366150
0.043808002
0
$-0.058329007$
3.765486273
0.157206014
$\mathrm{H}$
3.019952218
$-0.148473012$
$-0.034170002$

LIIC path connecting the ${ }^{1} \pi \pi^{*}$ and the ${ }^{1} \pi m^{*} / 1 n \pi^{*}$ minima of alloxazine (10 points)

\section{Coord.1}

$\begin{array}{llll}\text { C } & -3.366623240 & -1.821869129 & 0.012994000 \\ \text { C } & -2.905464207 & -0.454858035 & 0.111924010 \\ \text { C } & -1.556781114 & -0.110959007 & 0.054358002 \\ \text { C } & -0.609258044 & -1.191147084 & -0.061409004 \\ \text { C } & -1.073403077 & -2.531698182 & -0.171381011 \\ \text { C } & -2.455319178 & -2.850572206 & -0.138876012 \\ \text { C } & 1.050916076 & 0.317987022 & -0.001113997 \\ \text { C } & 0.117653010 & 1.408876100 & 0.069253003 \\ \text { C } & 0.605132044 & 2.775843197 & 0.110703007 \\ \text { C } & 2.920340210 & 1.876944134 & 0.048095005 \\ \text { H } & -3.616115258 & 0.338461022 & 0.235474017 \\ \text { H } & -0.333904023 & -3.300318235 & -0.282962018 \\ \text { H } & -2.772337197 & -3.869035280 & -0.235136016 \\ \text { H } & 2.379553172 & 3.800976276 & 0.109477009\end{array}$




$\begin{array}{llll}\mathrm{H} & -4.422675319 & -2.009574146 & 0.051174001 \\ \mathrm{~N} & 0.725505051 & -0.938229066 & -0.070391004 \\ \mathrm{~N} & -1.205819089 & 1.161337084 & 0.106935006 \\ \mathrm{~N} & 2.372156168 & 0.610219046 & 0.010946000 \\ \mathrm{~N} & 2.002862147 & 2.880174207 & 0.088321006 \\ \mathrm{O} & 4.099937296 & 2.048006147 & 0.044341005 \\ \mathrm{O} & -0.058438007 & 3.766582273 & 0.156497012 \\ \mathrm{H} & 3.017187219 & -0.150336013 & -0.033619001\end{array}$

\section{Coord.2}

C

$-3.367188242$

$-1.820536131$

0.012963001

C

$-2.909867210$

$-0.456927033$

0.111321007

C

$-1.562632111$

$-0.114754007$

0.054467002

C

$-0.611357042$

$-1.191898087$

$-0.061507002$

C

$-1.074377075$

$-2.531992182$

$-0.171030013$

C

$-2.454012178$

$-2.849639203$

$-0.138612011$

C

1.050325075

0.319184021

$-0.001254002$

C

0.120392010

1.409910101

0.069790007

C

0.606770043

2.776786202

0.111592009

C

2.921102209

1.876629136

0.047830003

H $\quad-3.621293262$

0.335832022

0.234018018

H

$-0.334535025$

$-3.300399237$

$-0.282336023$ 


$\begin{array}{lrrr}\mathrm{H} & -2.771697199 & -3.867980281 & -0.234341017 \\ \mathrm{H} & 2.381111170 & 3.801201271 & 0.110583006 \\ \mathrm{H} & -4.422729317 & -2.011304143 & 0.051212001 \\ \mathrm{~N} & 0.722726050 & -0.936573070 & -0.071020005 \\ \mathrm{~N} & -1.202911085 & 1.157420083 & 0.106544008 \\ \mathrm{~N} & 2.372978171 & 0.610736042 & 0.010166003 \\ \mathrm{~N} & 2.003501144 & 2.880771209 & 0.089043005 \\ \mathrm{O} & 4.100519296 & 2.049023146 & 0.043860004 \\ \mathrm{O} & -0.059227005 & 3.766028272 & 0.157787013 \\ \mathrm{H} & 3.017520220 & -0.150117013 & -0.035177005\end{array}$

\section{Coord.3}

$\begin{array}{llll}\text { C } & -3.367753245 & -1.819201133 & 0.012931002 \\ \text { C } & -2.914269208 & -0.458996032 & 0.110717009 \\ \text { C } & -1.568483112 & -0.118549007 & 0.054576002 \\ \text { C } & -0.613456044 & -1.192648084 & -0.061604005 \\ \text { C } & -1.075350078 & -2.532286183 & -0.170680010 \\ \text { C } & -2.452703174 & -2.848705205 & -0.138348010 \\ \text { C } & 1.049734074 & 0.320381025 & -0.001394001 \\ \text { C } & 0.123131010 & 1.410944103 & 0.070328006 \\ \text { C } & 0.608408043 & 2.777729201 & 0.112482006 \\ \text { C } & 2.921864209 & 1.876314133 & 0.047565002\end{array}$




$\begin{array}{llll}\mathrm{H} & -3.626470262 & 0.333202022 & 0.232562018 \\ \mathrm{H} & -0.335165026 & -3.300479238 & -0.281710022 \\ \mathrm{H} & -2.771056202 & -3.866924276 & -0.233547018 \\ \mathrm{H} & 2.382668174 & 3.801425272 & 0.111689007 \\ \mathrm{H} & -4.422781319 & -2.013033145 & 0.051249001 \\ \mathrm{~N} & 0.719946049 & -0.934917068 & -0.071648007 \\ \mathrm{~N} & -1.200002087 & 1.153503081 & 0.106153009 \\ \mathrm{~N} & 2.373799168 & 0.611252042 & 0.009386001 \\ \mathrm{~N} & 2.004139147 & 2.881367205 & 0.089766009 \\ \mathrm{O} & 4.101101296 & 2.050039145 & 0.043380004 \\ \mathrm{O} & -0.060015003 & 3.765473271 & 0.159077010 \\ \mathrm{H} & 3.017851215 & -0.149897012 & -0.036735003\end{array}$

\section{Coord. 4}

C

$-3.368317242$

$-1.817867130$

0.012900003

C

$-2.918672211$

$-0.461065035$

0.110114007

C

$-1.574334114$

$-0.122343007$

0.054685002

C

$$
-0.615554042
$$

$-1.193399087$

$-0.061702004$

C

$-1.076324077$

$-2.532580183$

$-0.170329012$

C

$-2.451395175$

$-2.847770207$

$-0.138084009$

C

1.049143078

0.321578024

$-0.001533000$

C

0.125870010

1.411978100

0.070865004 


$\begin{array}{llll}\mathrm{C} & 0.610046042 & 2.778672200 & 0.113371008 \\ \mathrm{C} & 2.922625208 & 1.875999135 & 0.047301006 \\ \mathrm{H} & -3.631647261 & 0.330573022 & 0.231106019 \\ \mathrm{H} & -0.335795022 & -3.300559239 & -0.281084021 \\ \mathrm{H} & -2.770415199 & -3.865868276 & -0.232752014 \\ \mathrm{H} & 2.384225172 & 3.801649273 & 0.112796009 \\ \mathrm{H} & -4.422834316 & -2.014762147 & 0.051287002 \\ \mathrm{~N} & 0.717167054 & -0.933261066 & -0.072277008 \\ \mathrm{~N} & -1.197094089 & 1.1495860085 & 0.105762005 \\ \mathrm{~N} & 2.374620171 & 0.611769043 & 0.008605999 \\ \mathrm{~N} & 2.004777144 & 2.881964207 & 0.090488008 \\ \mathrm{O} & 4.101682295 & 2.051055149 & 0.0429000003 \\ \mathrm{O} & -0.060804006 & 3.764917270 & 0.160367012 \\ \mathrm{H} & 3.018183215 & -0.149677012 & -0.038293001\end{array}$

\section{Coord.5}

$\begin{array}{llll}\text { C } & -3.368882245 & -1.816534133 & 0.012868003 \\ \text { C } & -2.923074209 & -0.463134034 & 0.109511009 \\ \text { C } & -1.580185116 & -0.126138007 & 0.054794002 \\ \text { C } & -0.617653044 & -1.194149085 & -0.061799002 \\ \text { C } & -1.077297075 & -2.532874183 & -0.169978014 \\ \text { C } & -2.450087176 & -2.846836204 & -0.137821008\end{array}$




$\begin{array}{llll}\mathrm{C} & 1.048552077 & 0.322775023 & -0.001672999 \\ \mathrm{C} & 0.128609011 & 1.413012101 & 0.071402003 \\ \mathrm{C} & 0.611684041 & 2.779614199 & 0.114261010 \\ \mathrm{C} & 2.923387212 & 1.875684137 & 0.047036004 \\ \mathrm{H} & -3.636824260 & 0.327943021 & 0.229650014 \\ \mathrm{H} & -0.336426024 & -3.300639240 & -0.280458020 \\ \mathrm{H} & -2.769773201 & -3.864812277 & -0.231957015 \\ \mathrm{H} & 2.385782170 & 3.801873273 & 0.113902011 \\ \mathrm{H} & -4.422886318 & -2.016491144 & 0.051325002 \\ \mathrm{~N} & 0.714387053 & -0.931605069 & -0.072906004 \\ \mathrm{~N} & -1.194185085 & 1.145668083 & 0.105371007 \\ \mathrm{~N} & 2.375441174 & 0.612286044 & 0.007826002 \\ \mathrm{~N} & 2.005415146 & 2.882560209 & 0.091211007 \\ \mathrm{O} & 4.102263295 & 2.052071149 & 0.042419002 \\ \mathrm{O} & -0.061592004 & 3.764362269 & 0.161657014 \\ \mathrm{H} & 3.018514216 & -0.149458012 & -0.039851005\end{array}$

\section{Coord.6}

$\begin{array}{llll}\text { C } & -3.369446242 & -1.815200130 & 0.012836999\end{array}$

$\begin{array}{llll}\text { C } & -2.927476213 & -0.465203032 & 0.108907006\end{array}$

$\begin{array}{llll}\text { C } & -1.586036112 & -0.129933007 & 0.054903002\end{array}$

$\begin{array}{llll}\text { C } & -0.619752047 & -1.194900088 & -0.061897006\end{array}$ 


$\begin{array}{lrrr}\text { C } & -1.078271079 & -2.533168184 & -0.169627010 \\ \text { C } & -2.448779176 & -2.845902206 & -0.137557012 \\ \text { C } & 1.047960076 & 0.323972022 & -0.001812998 \\ \text { C } & 0.131349011 & 1.414046103 & 0.071939006 \\ \text { C } & 0.613322046 & 2.780557198 & 0.115150007 \\ \text { C } & 2.924148212 & 1.875369134 & 0.046771003 \\ \text { H } & -3.642001265 & 0.325314021 & 0.228194015 \\ \text { H } & -0.337056025 & -3.300719235 & -0.279832020 \\ \text { H } & -2.769132198 & -3.863756277 & -0.231162016 \\ \text { H } & 2.387339174 & 3.802097274 & 0.115008007 \\ \text { H } & -4.422939321 & -2.018220146 & 0.051362002 \\ \mathrm{~N} & 0.711607052 & -0.929949067 & -0.073535005 \\ \mathrm{~N} & -1.191277087 & 1.141751081 & 0.104980008 \\ \mathrm{~N} & 2.376262171 & 0.612802045 & 0.007045000 \\ \mathrm{~N} & 2.006053144 & 2.883156205 & 0.091933006 \\ \mathrm{O} & 4.102844294 & 2.053088148 & 0.041938001 \\ \mathrm{O} & -0.062381007 & 3.763807273 & 0.162947010 \\ \mathrm{H} & 3.018846216 & -0.149238012 & -0.041409003\end{array}$

\section{Coord. 7}

$\begin{array}{llll}\text { C } & -3.370011244 & -1.813865132 & 0.012805999 \\ \text { C } & -2.931879211 & -0.467272036 & 0.108304009\end{array}$




\begin{tabular}{|c|c|c|c|}
\hline C & -1.591887114 & -0.133728012 & 0.055012002 \\
\hline C & -0.621850044 & -1.195650086 & -0.061994004 \\
\hline C & -1.079244077 & -2.533461184 & -0.169277013 \\
\hline C & -2.447471177 & -2.844968203 & -0.137293011 \\
\hline C & 1.047369075 & 0.325170021 & -0.001953003 \\
\hline C & 0.134088011 & 1.415080100 & 0.072477005 \\
\hline C & 0.614960046 & 2.781499202 & 0.116039008 \\
\hline C & 2.924910211 & 1.875054136 & 0.046506002 \\
\hline $\mathrm{H}$ & -3.647178264 & 0.322684021 & 0.226738015 \\
\hline $\mathrm{H}$ & -0.337686026 & -3.300800236 & -0.279206019 \\
\hline $\mathrm{H}$ & -2.768491200 & -3.862700277 & -0.230368018 \\
\hline $\mathrm{H}$ & 2.388897172 & 3.802321275 & 0.116114009 \\
\hline $\mathrm{H}$ & -4.422991318 & -2.019949148 & 0.051400002 \\
\hline $\mathrm{N}$ & 0.708828051 & -0.928293065 & -0.074163006 \\
\hline $\mathrm{N}$ & -1.188368088 & 1.137834084 & 0.104589010 \\
\hline $\mathrm{N}$ & 2.377083174 & 0.613319046 & 0.006264998 \\
\hline $\mathrm{N}$ & 2.006691146 & 2.883752207 & 0.092655005 \\
\hline 0 & 4.103426294 & 2.054104147 & 0.041458005 \\
\hline 0 & -0.063169005 & 3.763251272 & 0.164237012 \\
\hline $\mathrm{H}$ & 3.019178217 & -0.149018012 & -0.042967002 \\
\hline
\end{tabular}

\section{Coord. 8}




\begin{tabular}{|c|c|c|c|}
\hline C & -3.370575241 & -1.812531129 & 0.012774000 \\
\hline C & -2.936281209 & -0.469341034 & 0.107701006 \\
\hline C & -1.597738116 & -0.137522012 & 0.055121002 \\
\hline C & -0.623949047 & -1.196401089 & -0.062092002 \\
\hline C & -1.080218075 & -2.533755184 & -0.168926015 \\
\hline C & -2.446163178 & -2.844034205 & -0.137029009 \\
\hline C & 1.046778074 & 0.326367026 & -0.002093002 \\
\hline C & 0.136827012 & 1.416114101 & 0.073014004 \\
\hline C & 0.616598045 & 2.782442202 & 0.116929011 \\
\hline C & 2.925671210 & 1.874739138 & 0.046241006 \\
\hline $\mathrm{H}$ & -3.652355263 & 0.320055020 & 0.225282016 \\
\hline $\mathrm{H}$ & -0.338317023 & -3.300880238 & -0.278580018 \\
\hline $\mathrm{H}$ & -2.767850197 & -3.861644278 & -0.229573019 \\
\hline $\mathrm{H}$ & 2.390454170 & 3.802545276 & 0.117221011 \\
\hline $\mathrm{H}$ & -4.423044320 & -2.021678144 & 0.051437002 \\
\hline $\mathrm{N}$ & 0.706048050 & -0.926637068 & -0.074792007 \\
\hline $\mathrm{N}$ & -1.185459084 & 1.133916083 & 0.104198006 \\
\hline $\mathrm{N}$ & 2.377904171 & 0.613836047 & 0.005485002 \\
\hline $\mathrm{N}$ & 2.007329143 & 2.884349209 & 0.093378009 \\
\hline 0 & 4.104007294 & 2.055120146 & 0.040978004 \\
\hline 0 & -0.063958003 & 3.762696271 & 0.165527014 \\
\hline $\mathrm{H}$ & 3.019509217 & -0.148799012 & -0.044525005 \\
\hline
\end{tabular}




\section{Coord.9}

C

$-3.371140244$

$-1.811198132$

0.012743001

C

$-2.940683212$

$-0.471410033$

0.107097008

C

$-1.603589117$

$-0.141317012$

0.055230002

C

$-0.626048045$

$-1.197151086$

$-0.062189006$

C

$-1.081192079$

$-2.534049184$

$-0.168575011$

C

$-2.444855174$

$-2.843100202$

$-0.136765008$

C

1.046187078

0.327564024

$-0.002232001$

C

0.139566012

1.417148103

0.073551007

C

0.618236044

2.783384201

0.117818007

C

2.926433210

1.874424134

0.045976004

$\mathrm{H}$

$-3.657532262$

0.317425025

0.223827016

$\mathrm{H}$

$-0.338947024$

$-3.300960239$

$-0.277954023$

$\mathrm{H}$

$-2.767209199$

$-3.860588278$

$-0.228778015$

$\mathrm{H}$

2.392011174

3.802769271

0.118327007

$\mathrm{H}$

$-4.423096317$

$-2.023407146$

0.051475003

$\mathrm{N}$

0.703269050

$-0.924981066$

$-0.075421003$

$\mathrm{N}$

$-1.182551086$

1.129999081

0.103807008

$\mathrm{N}$

2.378725173

0.614352042

0.004705000

N

2.007967146

2.884945205

0.094100008

0

4.104588293

2.056136145

0.040497004 

0
$-0.064746006$
3.762141270
0.166817010
$\begin{array}{ll}\text { H } & 3.019841217\end{array}$
$-0.148579012$
$-0.046083004$

\section{Coord.10}

$\begin{array}{llll}\text { C } & -3.370573241 & -1.810395132 & 0.012910999 \\ \text { C } & -2.943959211 & -0.474008034 & 0.106671010 \\ \text { C } & -1.608315115 & -0.145637009 & 0.055509005 \\ \text { C } & -0.627018048 & -1.198425086 & -0.062100003 \\ \text { C } & -1.081032077 & -2.534869182 & -0.168015010 \\ \text { C } & -2.442412174 & -2.842696207 & -0.136285007 \\ \text { C } & 1.046718076 & 0.328244023 & -0.002211998 \\ \mathrm{C} & 0.143424010 & 1.417663104 & 0.074231005 \\ \mathrm{C} & 0.620988046 & 2.783810199 & 0.118827011 \\ \mathrm{C} & 2.928311212 & 1.873599136 & 0.045844001 \\ \mathrm{H} & -3.661585263 & 0.314266022 & 0.222535014 \\ \mathrm{H} & -0.338442025 & -3.301565236 & -0.277106021 \\ \mathrm{H} & -2.765430201 & -3.860065276 & -0.227750014 \\ \mathrm{H} & 2.394678174 & 3.802482272 & 0.119534007 \\ \mathrm{H} & -4.422016319 & -2.025671147 & 0.051717006 \\ \mathrm{~N} & 0.701616048 & -0.923844066 & -0.075868005 \\ \mathrm{~N} & -1.178522084 & 1.125559083 & 0.103565010 \\ \mathrm{~N} & 2.380667169 & 0.614357043 & 0.004078999\end{array}$



N
2.009718146
2.885029207
0.094939008
0
4.106285296
2.056647145
0.040145005
0
$-0.064424007$
3.761067273
0.168211012
H
3.021296217
$-0.148870012$
$-0.047475005$

LIIC path connecting the ${ }^{1} \pi \pi^{*}$ and the ${ }^{3} n \pi^{*}$ minima of alloxazine (10 points)

Coord.1

$\begin{array}{llll}\text { C } & -3.366623240 & -1.821869129 & 0.012994000 \\ \text { C } & -2.905464207 & -0.454858035 & 0.111924010 \\ \text { C } & -1.556781114 & -0.110959007 & 0.054358002 \\ \text { C } & -0.609258044 & -1.191147084 & -0.061409004 \\ \text { C } & -1.073403077 & -2.531698182 & -0.171381011 \\ \text { C } & -2.455319178 & -2.850572206 & -0.138876012 \\ \text { C } & 1.050916076 & 0.317987022 & -0.001113997 \\ \text { C } & 0.117653010 & 1.408876100 & 0.069253003 \\ \text { C } & 0.605132044 & 2.775843197 & 0.110703007 \\ \text { C } & 2.920340210 & 1.876944134 & 0.048095005 \\ \text { H } & -3.616115258 & 0.338461022 & 0.235474017 \\ \text { H } & -0.333904023 & -3.300318235 & -0.282962018 \\ \text { H } & -2.772337197 & -3.869035280 & -0.235136016 \\ \text { H } & 2.379553172 & 3.800976276 & 0.109477009\end{array}$




$\begin{array}{llll}\mathrm{H} & -4.422675319 & -2.009574146 & 0.051174001 \\ \mathrm{~N} & 0.725505051 & -0.938229066 & -0.070391004 \\ \mathrm{~N} & -1.205819089 & 1.161337084 & 0.106935006 \\ \mathrm{~N} & 2.372156168 & 0.610219046 & 0.010946000 \\ \mathrm{~N} & 2.002862147 & 2.880174207 & 0.088321006 \\ \mathrm{O} & 4.099937296 & 2.048006147 & 0.044341005 \\ \mathrm{O} & -0.058438007 & 3.766582273 & 0.156497012 \\ \mathrm{H} & 3.017187219 & -0.150336013 & -0.033619001\end{array}$

\section{Coord.2}

$\begin{array}{llll}\text { C } & -3.368673241 & -1.819355129 & 0.013071001 \\ \text { C } & -2.916090207 & -0.459213032 & 0.111081009 \\ \text { C } & -1.565047112 & -0.119043010 & 0.054400003 \\ \text { C } & -0.614114045 & -1.191559085 & -0.061118004 \\ \text { C } & -1.076283076 & -2.530610183 & -0.170001012 \\ \text { C } & -2.452467177 & -2.847361206 & -0.137855007 \\ \text { C } & 1.052336075 & 0.317783024 & -0.001594998 \\ \text { C } & 0.124317008 & 1.410613103 & 0.070139005 \\ \text { C } & 0.608782044 & 2.777830200 & 0.112299006 \\ \text { C } & 2.923136208 & 1.876886137 & 0.047630006 \\ \text { H } & -3.627763259 & 0.333371025 & 0.233414015 \\ \text { H } & -0.336344022 & -3.299084236 & -0.280738019\end{array}$




$\begin{array}{lrrr}\mathrm{H} & -2.769428198 & -3.866147280 & -0.233161015 \\ \mathrm{H} & 2.382304174 & 3.801943273 & 0.110938010 \\ \mathrm{H} & -4.423511319 & -2.013762145 & 0.050867004 \\ \mathrm{~N} & 0.723131051 & -0.936722065 & -0.071220003 \\ \mathrm{~N} & -1.1968480085 & 1.151570081 & 0.106184008 \\ \mathrm{~N} & 2.376862173 & 0.611449044 & 0.009809999 \\ \mathrm{~N} & 2.004491145 & 2.881583210 & 0.089317007 \\ \mathrm{O} & 4.102116295 & 2.052486145 & 0.043647005 \\ \mathrm{O} & -0.059661004 & 3.765392270 & 0.158973011 \\ \mathrm{H} & 3.022177217 & -0.148593009 & -0.035713003\end{array}$

\section{Coord.3}

$\begin{array}{llll}\text { C } & -3.370723242 & -1.816840130 & 0.013149002 \\ \text { C } & -2.926715208 & -0.463567033 & 0.110239009 \\ \text { C } & -1.573312114 & -0.127127008 & 0.054442004 \\ \text { C } & -0.618969045 & -1.191971087 & -0.060828004 \\ \text { C } & -1.079162076 & -2.529521184 & -0.168621013 \\ \text { C } & -2.449613176 & -2.844148206 & -0.136834007 \\ \text { C } & 1.053755075 & 0.317579021 & -0.002074999 \\ \text { C } & 0.130981011 & 1.412349100 & 0.071025006 \\ \text { C } & 0.612431044 & 2.779817202 & 0.113894010 \\ \text { C } & 2.925931211 & 1.876828134 & 0.047164002\end{array}$




$\begin{array}{llll}\mathrm{H} & -3.639409264 & 0.328280022 & 0.231354018 \\ \mathrm{H} & -0.338784027 & -3.297850237 & -0.278514019 \\ \mathrm{H} & -2.766519200 & -3.863258279 & -0.231186015 \\ \mathrm{H} & 2.385054170 & 3.802908275 & 0.112399010 \\ \mathrm{H} & -4.424346318 & -2.017950144 & 0.050559002 \\ \mathrm{~N} & 0.720756050 & -0.935214068 & -0.072049006 \\ \mathrm{~N} & -1.187877086 & 1.141802083 & 0.105433005 \\ \mathrm{~N} & 2.381567173 & 0.612680043 & 0.008674998 \\ \mathrm{~N} & 2.006119143 & 2.882992208 & 0.090313009 \\ \mathrm{O} & 4.104294293 & 2.056965149 & 0.042952005 \\ \mathrm{O} & -0.060885007 & 3.764200272 & 0.161448010 \\ \mathrm{H} & 3.027167216 & -0.146850010 & -0.037806005\end{array}$

\section{Coord.4}

$\begin{array}{llll}\text { C } & -3.372772242 & -1.814325130 & 0.013227002 \\ \text { C } & -2.937341209 & -0.467922035 & 0.109396008 \\ \text { C } & -1.581577111 & -0.135211010 & 0.054484005 \\ \text { C } & -0.623825045 & -1.192383088 & -0.060537004 \\ \text { C } & -1.082042080 & -2.528433180 & -0.167240014 \\ \text { C } & -2.446760175 & -2.840936206 & -0.135813008 \\ \text { C } & 1.055175074 & 0.317374023 & -0.002556000 \\ \text { C } & 0.137645009 & 1.414085103 & 0.071912008\end{array}$




$\begin{array}{llll}\mathrm{C} & 0.616081044 & 2.781804199 & 0.115490008 \\ \mathrm{C} & 2.928726209 & 1.876770136 & 0.046699003 \\ \mathrm{H} & -3.651056265 & 0.323190025 & 0.229294015 \\ \mathrm{H} & -0.341224026 & -3.296615238 & -0.276290019 \\ \mathrm{H} & -2.763609201 & -3.860369278 & -0.229210019 \\ \mathrm{H} & 2.387804172 & 3.803874272 & 0.113860010 \\ \mathrm{H} & -4.425181318 & -2.022137147 & 0.050252005 \\ \mathrm{~N} & 0.718382050 & -0.933707067 & -0.072878005 \\ \mathrm{~N} & -1.178907087 & 1.132035080 & 0.104683008 \\ \mathrm{~N} & 2.386273173 & 0.613910047 & 0.007539003 \\ \mathrm{~N} & 2.007748146 & 2.884400206 & 0.091309005 \\ \mathrm{O} & 4.106472296 & 2.061445148 & 0.042258005 \\ \mathrm{O} & -0.062108004 & 3.763009269 & 0.163924014 \\ \mathrm{H} & 3.032156220 & -0.145107011 & -0.0399000002\end{array}$

\section{Coord.5}

$\begin{array}{llll}C & -3.374821243 & -1.811810131 & 0.013304003 \\ C & -2.947966210 & -0.472276037 & 0.108554008 \\ C & -1.589842114 & -0.143295013 & 0.054526006 \\ C & -0.628680045 & -1.192795084 & -0.060246004 \\ C & -1.084921080 & -2.527344181 & -0.165860015 \\ \text { C } & -2.443907174 & -2.837724206 & -0.134792008\end{array}$




$\begin{array}{llll}\mathrm{C} & 1.056594073 & 0.317170020 & -0.003037001 \\ \mathrm{C} & 0.144309012 & 1.415821101 & 0.072798004 \\ \mathrm{C} & 0.619730044 & 2.783791201 & 0.117086007 \\ \mathrm{C} & 2.931521212 & 1.876712133 & 0.046233004 \\ \mathrm{H} & -3.662702266 & 0.318100023 & 0.227234018 \\ \mathrm{H} & -0.343664025 & -3.295381239 & -0.274066020 \\ \mathrm{H} & -2.760700198 & -3.857480277 & -0.227235019 \\ \mathrm{H} & 2.390554174 & 3.804839275 & 0.115320010 \\ \mathrm{H} & -4.426016317 & -2.026325146 & 0.049945003 \\ \mathrm{~N} & 0.716007050 & -0.932199065 & -0.073707003 \\ \mathrm{~N} & -1.169936082 & 1.122267081 & 0.103932010 \\ \mathrm{~N} & 2.390978172 & 0.615141045 & 0.006404003 \\ \mathrm{~N} & 2.009376144 & 2.885809209 & 0.092305007 \\ \mathrm{O} & 4.108650295 & 2.065924146 & 0.041564005 \\ \mathrm{O} & -0.063331007 & 3.761817270 & 0.166400013 \\ \mathrm{H} & 3.037146218 & -0.143364012 & -0.041994004\end{array}$

\section{Coord.6}

C

$-3.376871244$

$-1.809295131$

0.013381998

C

$-2.958591210$

$-0.476631033$

0.107712008

C

$-1.598107116$

$-0.151379010$

0.054568006

C

$-0.633536046$

$-1.193207086$

$-0.059956005$ 


\begin{tabular}{|c|c|c|c|}
\hline C & -1.087801079 & -2.526255181 & -0.164480010 \\
\hline C & -2.441054178 & -2.834512206 & -0.133771008 \\
\hline C & 1.058013078 & 0.316966022 & -0.003517002 \\
\hline C & 0.150973010 & 1.417557104 & 0.073684005 \\
\hline C & 0.623379044 & 2.785777198 & 0.118681011 \\
\hline C & 2.934316210 & 1.876654135 & 0.045768006 \\
\hline $\mathrm{H}$ & -3.674349266 & 0.313009020 & 0.225174016 \\
\hline $\mathrm{H}$ & -0.346104025 & -3.294146235 & -0.271841020 \\
\hline $\mathrm{H}$ & -2.757790199 & -3.854591276 & -0.225260018 \\
\hline $\mathrm{H}$ & 2.393304171 & 3.805805272 & 0.116781010 \\
\hline $\mathrm{H}$ & -4.426851316 & -2.030513145 & 0.049637006 \\
\hline $\mathrm{N}$ & 0.713633050 & -0.930691069 & -0.074536007 \\
\hline $\mathrm{N}$ & -1.160964083 & 1.112499078 & 0.103181007 \\
\hline $\mathrm{N}$ & 2.395683172 & 0.616371044 & 0.005268002 \\
\hline $\mathrm{N}$ & 2.011004147 & 2.887217207 & 0.093302008 \\
\hline 0 & 4.110827298 & 2.070403150 & 0.040870005 \\
\hline 0 & -0.064555005 & 3.760626272 & 0.168875013 \\
\hline $\mathrm{H}$ & 3.042135217 & -0.141622008 & -0.044087005 \\
\hline
\end{tabular}

\section{Coord. 7}

$\begin{array}{llll}\text { C } & -3.378920245 & -1.806780131 & 0.013458999 \\ \text { C } & -2.969217211 & -0.480985035 & 0.106869007\end{array}$




\begin{tabular}{|c|c|c|c|}
\hline C & -1.606372113 & -0.159463013 & 0.054610002 \\
\hline C & -0.638391046 & -1.193619087 & -0.059665005 \\
\hline C & -1.090680079 & -2.525166182 & -0.163099011 \\
\hline C & -2.438201177 & -2.831299205 & -0.132750008 \\
\hline C & 1.059433078 & 0.316762025 & -0.003998003 \\
\hline C & 0.157637013 & 1.419293102 & 0.074570007 \\
\hline C & 0.627029044 & 2.787764201 & 0.120277009 \\
\hline C & 2.937111213 & 1.876596137 & 0.045302002 \\
\hline $\mathrm{H}$ & -3.685996267 & 0.307919023 & 0.223114014 \\
\hline $\mathrm{H}$ & -0.348544024 & -3.292912236 & -0.269617020 \\
\hline $\mathrm{H}$ & -2.754880200 & -3.851702275 & -0.223284017 \\
\hline $\mathrm{H}$ & 2.396054173 & 3.806770274 & 0.118242010 \\
\hline $\mathrm{H}$ & -4.427686321 & -2.034700149 & 0.049330004 \\
\hline $\mathrm{N}$ & 0.711258049 & -0.929184067 & -0.075365006 \\
\hline $\mathrm{N}$ & -1.151994084 & 1.102732080 & 0.102430009 \\
\hline$N$ & 2.400388171 & 0.617601042 & 0.004133001 \\
\hline$N$ & 2.012633145 & 2.8886626210 & 0.094298005 \\
\hline 0 & 4.113005297 & 2.074883149 & 0.040175004 \\
\hline 0 & -0.065778007 & 3.759434269 & 0.171351012 \\
\hline $\mathrm{H}$ & 3.047124221 & -0.139879010 & -0.046181002 \\
\hline
\end{tabular}

\section{Coord.8}




$\begin{array}{llll}\mathrm{C} & -3.380969246 & -1.804265132 & 0.013537000 \\ \mathrm{C} & -2.979842212 & -0.485340036 & 0.106026007 \\ \mathrm{C} & -1.614638116 & -0.167546010 & 0.054652003 \\ \mathrm{C} & -0.643247046 & -1.194031084 & -0.059374005 \\ \mathrm{C} & -1.093560078 & -2.524078183 & -0.161719012 \\ \mathrm{C} & -2.435348176 & -2.828087205 & -0.131729008 \\ \mathrm{C} & 1.060852077 & 0.316557021 & -0.004478999 \\ \mathrm{C} & 0.164300011 & 1.421030100 & 0.075456003 \\ \mathrm{C} & 0.630678044 & 2.789751203 & 0.121873008 \\ \mathrm{C} & 2.939906211 & 1.876538134 & 0.044837003 \\ \mathrm{H} & -3.697642268 & 0.302829020 & 0.221054017 \\ \mathrm{H} & -0.350984023 & -3.291677237 & -0.267393020 \\ \mathrm{H} & -2.751971197 & -3.848813274 & -0.221309017 \\ \mathrm{H} & 2.398804174 & 3.807736272 & 0.119703011 \\ \mathrm{H} & -4.428521320 & -2.038888147 & 0.049023002 \\ \mathrm{~N} & 0.708883049 & -0.927676066 & -0.076194004 \\ \mathrm{~N} & -1.143023080 & 1.092964077 & 0.101679006 \\ \mathrm{~N} & 2.405094171 & 0.618832046 & 0.002997001 \\ \mathrm{~N} & 2.014261143 & 2.890034208 & 0.095294006 \\ \mathrm{O} & 3.115183295 & 2.079362148 & 0.039481004 \\ \mathrm{~N} & -0.067002005 & 3.758243271 & 0.173827011 \\ \mathrm{~N} & 3.052114219 & -0.138136011 & -0.048275004\end{array}$




\section{Coord.9}

\begin{tabular}{|c|c|c|c|}
\hline C & -3.383019241 & -1.801750127 & 0.013614000 \\
\hline C & -2.990467213 & -0.489694033 & 0.105184006 \\
\hline C & -1.622903119 & -0.175630013 & 0.054694004 \\
\hline C & -0.648102046 & -1.194443085 & -0.059084005 \\
\hline C & -1.096439078 & -2.522989184 & -0.160339013 \\
\hline C & -2.432495175 & -2.824875205 & -0.130709008 \\
\hline$C$ & 1.062272077 & 0.316353023 & -0.004958999 \\
\hline C & 0.170964014 & 1.422766103 & 0.076343005 \\
\hline C & 0.634328044 & 2.791737200 & 0.123468011 \\
\hline 2 & 2.942700213 & 1.876480136 & 0.044372004 \\
\hline$\dashv$ & -3.709289269 & 0.297738023 & 0.218994014 \\
\hline $\mathrm{H}$ & -0.353424028 & -3.290443238 & -0.265169021 \\
\hline $\mathrm{H}$ & -2.749061198 & -3.845924279 & -0.219334016 \\
\hline $\mathrm{H}$ & 2.401554171 & 3.808701274 & 0.121164011 \\
\hline $\mathrm{H}$ & -4.429356320 & -2.043075146 & 0.048715004 \\
\hline$N$ & 0.706509048 & -0.926169069 & -0.077024008 \\
\hline$\sqrt{ }$ & -1.134052081 & 1.083196078 & 0.100929008 \\
\hline$\sqrt{ }$ & 2.409799176 & 0.620062044 & 0.001862000 \\
\hline | & 2.015890147 & 2.891443206 & 0.096290008 \\
\hline O & 4.117361298 & 2.083841151 & 0.038787004 \\
\hline
\end{tabular}



0
$-0.068225002$
3.757051272
0.176303010
$\begin{array}{ll}H & 3.057103218\end{array}$
$-0.136393012$
$-0.050368006$

\section{Coord.10}

$\begin{array}{llll}\text { C } & -3.383845245 & -1.799789128 & 0.013881002 \\ \text { C } & -2.999876217 & -0.494599035 & 0.104505008 \\ \text { C } & -1.629953119 & -0.184259015 & 0.054892006 \\ \text { C } & -0.651738044 & -1.195397086 & -0.058618006 \\ \text { C } & -1.098093079 & -2.522446179 & -0.158757011 \\ \text { C } & -2.428414176 & -2.822214200 & -0.129479010 \\ \text { C } & 1.064903077 & 0.315616022 & -0.005296001 \\ \mathrm{C} & 0.178835012 & 1.423966102 & 0.077352008 \\ \mathrm{C} & 0.639177049 & 2.793191199 & 0.125160008 \\ \mathrm{C} & 2.946699211 & 1.875898136 & 0.044018001 \\ \mathrm{H} & -3.719722268 & 0.292096020 & 0.217083018 \\ \mathrm{H} & -0.354634023 & -3.289752238 & -0.262729016 \\ \mathrm{H} & -2.744919195 & -3.843590279 & -0.217130014 \\ \mathrm{H} & 2.405498171 & 3.809142274 & 0.122699011 \\ \mathrm{H} & -4.428966321 & -2.047821146 & 0.048603004 \\ \mathrm{~N} & 0.705352050 & -0.925197066 & -0.077684003 \\ \mathrm{~N} & -1.123872081 & 1.072887076 & 0.100309008 \\ \mathrm{~N} & 2.415714176 & 0.620766046 & 0.000862998\end{array}$




$\begin{array}{lrrr}\mathrm{N} & 2.018717144 & 2.892324206 & 0.097379007 \\ \mathrm{O} & 4.120741296 & 2.087803149 & 0.038199004 \\ \mathrm{O} & -0.068253006 & 3.755324271 & 0.178856015 \\ \mathrm{H} & 3.063306223 & -0.135174010 & -0.052311002\end{array}$

LIIC path connecting the ${ }^{3} n \pi^{*}$ and the ${ }^{3} n \pi^{*} / 3 \pi \pi^{*}$ minima of alloxazine (10 points)

\section{Coord.1}

$\begin{array}{lrrr}\text { C } & -3.383845245 & -1.799789128 & 0.013881002 \\ \text { C } & -2.999876217 & -0.494599035 & 0.104505008 \\ \text { C } & -1.629953119 & -0.184259015 & 0.054892006 \\ \text { C } & -0.651738044 & -1.195397086 & -0.058618006 \\ \text { C } & -1.098093079 & -2.522446179 & -0.158757011 \\ \text { C } & -2.428414176 & -2.822214200 & -0.129479010 \\ \text { C } & 1.064903077 & 0.315616022 & -0.005296001 \\ \text { C } & 0.178835012 & 1.423966102 & 0.077352008 \\ \text { C } & 0.639177049 & 2.793191199 & 0.125160008 \\ \text { C } & 2.946699211 & 1.875898136 & 0.044018001 \\ \text { H } & -3.719722268 & 0.292096020 & 0.217083018 \\ \text { H } & -0.354634023 & -3.289752238 & -0.262729016 \\ \text { H } & -2.744919195 & -3.843590279 & -0.217130014\end{array}$




$\begin{array}{lrrr}\mathrm{H} & 2.405498171 & 3.809142274 & 0.122699011 \\ \mathrm{H} & -4.428966321 & -2.047821146 & 0.048603004 \\ \mathrm{~N} & 0.705352050 & -0.925197066 & -0.077684003 \\ \mathrm{~N} & -1.123872081 & 1.072887076 & 0.100309008 \\ \mathrm{~N} & 2.415714176 & 0.620766046 & 0.000862998 \\ \mathrm{~N} & 2.018717144 & 2.892324206 & 0.097379007 \\ \mathrm{O} & 4.120741296 & 2.087803149 & 0.038199004 \\ \mathrm{O} & -0.068253006 & 3.755324271 & 0.178856015 \\ \mathrm{H} & 3.063306223 & -0.135174010 & -0.052311002\end{array}$

\section{Coord.2}

$\begin{array}{llll}\text { C } & -3.383806244 & -1.801275132 & 0.014987003 \\ \text { C } & -2.999615216 & -0.495033034 & 0.104879010 \\ \text { C } & -1.630900119 & -0.183311016 & 0.055311003 \\ \text { C } & -0.652490047 & -1.196486085 & -0.058195003 \\ \text { C } & -1.097057077 & -2.521042182 & -0.157575009 \\ \text { C } & -2.429536175 & -2.822421204 & -0.128176011 \\ \text { C } & 1.064618078 & 0.315510023 & -0.005352998 \\ \text { C } & 0.177517011 & 1.423082101 & 0.076989003 \\ \text { C } & 0.638943046 & 2.792934199 & 0.124480010 \\ \text { C } & 2.946548211 & 1.875686137 & 0.043704003 \\ \text { H } & -3.719986269 & 0.291323019 & 0.216734015\end{array}$




$\begin{array}{lrrr}\mathrm{H} & -0.353754028 & -3.288590239 & -0.260885019 \\ \mathrm{H} & -2.744737195 & -3.844144275 & -0.215631013 \\ \mathrm{H} & 2.405044174 & 3.808963275 & 0.121787011 \\ \mathrm{H} & -4.428999321 & -2.049053145 & 0.050117001 \\ \mathrm{~N} & 0.705525049 & -0.925484065 & -0.077296005 \\ \mathrm{~N} & -1.127199082 & 1.074589079 & 0.099823007 \\ \mathrm{~N} & 2.415631175 & 0.620671043 & 0.000802000 \\ \mathrm{~N} & 2.018373147 & 2.892026211 & 0.096783005 \\ \mathrm{O} & 4.120474295 & 2.087878149 & 0.037942003 \\ \mathrm{O} & -0.068293007 & 3.754865268 & 0.177709013 \\ \mathrm{H} & 3.063149221 & -0.135369012 & -0.051912002\end{array}$

\section{Coord. 3}

$\begin{array}{llll}\text { C } & -3.383766244 & -1.802761131 & 0.016092000 \\ \text { C } & -2.999353215 & -0.495467034 & 0.105253006 \\ \text { C } & -1.631846119 & -0.182363016 & 0.055731006 \\ \text { C } & -0.653243046 & -1.197574084 & -0.057771005 \\ \text { C } & -1.096021081 & -2.519636179 & -0.156392012 \\ \text { C } & -2.430657173 & -2.822627202 & -0.126873008 \\ \text { C } & 1.064332079 & 0.315405024 & -0.005411001 \\ \text { C } & 0.176200011 & 1.422197105 & 0.076627004 \\ \text { C } & 0.638709044 & 2.792676199 & 0.123800007\end{array}$




$\begin{array}{llll}\mathrm{C} & 2.946397210 & 1.875473133 & 0.043389005 \\ \mathrm{H} & -3.720248270 & 0.290551023 & 0.216385017 \\ \mathrm{H} & -0.352874027 & -3.287427234 & -0.259040021 \\ \mathrm{H} & -2.744554195 & -3.844697276 & -0.214132013 \\ \mathrm{H} & 2.404590172 & 3.808783275 & 0.120874011 \\ \mathrm{H} & -4.429031320 & -2.050285149 & 0.051631004 \\ \mathrm{~N} & 0.705698053 & -0.925770064 & -0.076908007 \\ \mathrm{~N} & -1.130527083 & 1.076291077 & 0.099338005 \\ \mathrm{~N} & 2.415548173 & 0.620577045 & 0.000741002 \\ \mathrm{~N} & 2.018028144 & 2.891727210 & 0.096187009 \\ \mathrm{O} & 4.120206298 & 2.087953150 & 0.037684003 \\ \mathrm{O} & -0.068333007 & 3.754405270 & 0.176562011 \\ \mathrm{H} & 3.062991219 & -0.135564009 & -0.051513003\end{array}$

\section{Coord. 4}

$\begin{array}{llll}\text { C } & -3.383726243 & -1.804247129 & 0.017198001 \\ \text { C } & -2.999092215 & -0.495901033 & 0.105628007 \\ \text { C } & -1.632793118 & -0.181415010 & 0.056151003 \\ \text { C } & -0.653995049 & -1.198662088 & -0.057347002 \\ \text { C } & -1.094984078 & -2.518231182 & -0.155210010 \\ \text { C } & -2.431778177 & -2.822834206 & -0.125570009 \\ \text { C } & 1.064047075 & 0.315299024 & -0.005467999\end{array}$




$\begin{array}{lrrr}\mathrm{C} & 0.174882010 & 1.421312104 & 0.076264004 \\ \mathrm{C} & 0.638475047 & 2.792419204 & 0.123121009 \\ \mathrm{C} & 2.946245214 & 1.875261134 & 0.043075002 \\ \mathrm{H} & -3.720511266 & 0.289778022 & 0.216036014 \\ \mathrm{H} & -0.351994027 & -3.286264235 & -0.257196018 \\ \mathrm{H} & -2.744371195 & -3.845250276 & -0.212632018 \\ \mathrm{H} & 2.404136175 & 3.808602275 & 0.119962011 \\ \mathrm{H} & -4.429063320 & -2.051517148 & 0.053145001 \\ \mathrm{~N} & 0.705870051 & -0.926056069 & -0.076520004 \\ \mathrm{~N} & -1.133854084 & 1.077992075 & 0.098852009 \\ \mathrm{~N} & 2.415464172 & 0.620482047 & 0.000679998 \\ \mathrm{~N} & 2.017683147 & 2.891428209 & 0.095590007 \\ \mathrm{O} & 4.119937296 & 2.088027150 & 0.037427003 \\ \mathrm{O} & -0.068372002 & 3.753946272 & 0.175415014 \\ \mathrm{H} & 3.062833223 & -0.135758010 & -0.051115003\end{array}$

\section{Coord.5}

C

$-3.383686243$

$-1.805733128$

0.018304003

C

$-2.998830214$

$-0.496334038$

0.106002008

C

$-1.633739118$

$-0.180466010$

0.056570006

C

$-0.654747047$

$-1.199751088$

$-0.056924004$

C $\quad-1.093948076$

$-2.516826179$

$-0.154028013$ 


$\begin{array}{lrrr}\text { C } & -2.432900176 & -2.823040204 & -0.124267011 \\ \text { C } & 1.063761075 & 0.315193025 & -0.005525002 \\ \text { C } & 0.173564015 & 1.420427102 & 0.075901004 \\ \text { C } & 0.638240045 & 2.792161203 & 0.122441011 \\ \text { C } & 2.946094213 & 1.875048135 & 0.042760004 \\ \text { H } & -3.720773267 & 0.289006022 & 0.215687016 \\ \text { H } & -0.351115026 & -3.285101236 & -0.255351021 \\ \text { H } & -2.744188195 & -3.845803277 & -0.211133017 \\ \text { H } & 2.403681173 & 3.808422276 & 0.119050011 \\ \text { H } & -4.429095319 & -2.052749146 & 0.054659004 \\ \mathrm{~N} & 0.706043050 & -0.926342068 & -0.076132006 \\ \mathrm{~N} & -1.137181080 & 1.079694079 & 0.098366007 \\ \mathrm{~N} & 2.415380176 & 0.620387044 & 0.000619000 \\ \mathrm{~N} & 2.017339145 & 2.891129208 & 0.094994005 \\ \mathrm{O} & 4.119669294 & 2.088102150 & 0.037169003 \\ \mathrm{O} & -0.068412003 & 3.753486269 & 0.174268011 \\ \mathrm{H} & 3.062675221 & -0.135953012 & -0.050716004\end{array}$

\section{Coord.6}

$\begin{array}{llll}\text { C } & -3.383646242 & -1.807218131 & 0.019408999 \\ \text { C } & -2.998568218 & -0.496768037 & 0.106376010 \\ \text { C } & -1.634685118 & -0.179518011 & 0.056990003\end{array}$




\begin{tabular}{|c|c|c|c|}
\hline C & -0.655499045 & -1.200839087 & -0.056500006 \\
\hline C & -1.092912080 & -2.515421182 & -0.152845011 \\
\hline C & -2.434021174 & -2.823247202 & -0.122964007 \\
\hline C & 1.063476077 & 0.315088025 & -0.005582000 \\
\hline C & 0.172246014 & 1.419542101 & 0.075538004 \\
\hline C & 0.638006048 & 2.791903203 & 0.121761007 \\
\hline C & 2.945943213 & 1.874835136 & 0.042446006 \\
\hline $\mathrm{H}$ & -3.721036268 & 0.288233021 & 0.215338013 \\
\hline $\mathrm{H}$ & -0.350235026 & -3.283938236 & -0.253507018 \\
\hline $\mathrm{H}$ & -2.744005195 & -3.846356278 & -0.209634017 \\
\hline $\mathrm{H}$ & 2.403227175 & 3.808242276 & 0.118138006 \\
\hline $\mathrm{H}$ & -4.429127318 & -2.053980150 & 0.056174007 \\
\hline $\mathrm{N}$ & 0.706216048 & -0.926629067 & -0.075743008 \\
\hline $\mathrm{N}$ & -1.140508081 & 1.081396077 & 0.097881006 \\
\hline $\mathrm{N}$ & 2.415297174 & 0.620293046 & 0.000558002 \\
\hline $\mathrm{N}$ & 2.016994143 & 2.890831207 & 0.094398009 \\
\hline 0 & 4.119401298 & 2.088177150 & 0.036912002 \\
\hline 0 & -0.068452004 & 3.753026271 & 0.173121014 \\
\hline $\mathrm{H}$ & 3.062517219 & -0.136148009 & -0.050317004 \\
\hline
\end{tabular}

\section{Coord.7}

$\begin{array}{llll}\text { C } & -3.383606242 & -1.808704130 & 0.020515001\end{array}$ 


$\begin{array}{llll}\mathrm{C} & -2.998306217 & -0.497202037 & 0.106750006 \\ \mathrm{C} & -1.635632117 & -0.178570011 & 0.057409006 \\ \mathrm{C} & -0.656251048 & -1.201927086 & -0.056077003 \\ \mathrm{C} & -1.091875077 & -2.514016179 & -0.151663009 \\ \mathrm{C} & -2.435142173 & -2.823453206 & -0.121661009 \\ \mathrm{C} & 1.063190077 & 0.314982020 & -0.005640003 \\ \mathrm{C} & 0.170928014 & 1.418657100 & 0.075176005 \\ \mathrm{C} & 0.637772046 & 2.791645202 & 0.121081009 \\ \mathrm{C} & 2.945791212 & 1.874623137 & 0.042132002 \\ \mathrm{H} & -3.721299269 & 0.287460020 & 0.214989016 \\ \mathrm{H} & -0.349355025 & -3.282775237 & -0.251662020 \\ \mathrm{H} & -2.743822195 & -3.846908279 & -0.208135017 \\ \mathrm{H} & 2.402773173 & 3.808062276 & 0.117225006 \\ \mathrm{H} & -4.429158317 & -2.055212149 & 0.057688004 \\ \mathrm{~N} & 0.706388052 & -0.926915066 & -0.075355004 \\ \mathrm{~N} & -1.143835081 & 1.083097080 & 0.097395009 \\ \mathrm{~N} & 2.415213172 & 0.620198043 & 0.000496998 \\ \mathrm{~N} & 2.016649145 & 2.890532206 & 0.093802007 \\ \mathrm{O} & 4.119133296 & 2.088252151 & 0.036654002 \\ \mathrm{H} & -0.068491004 & 3.752566268 & 0.171974012 \\ \mathrm{H} & 3.062359223 & -0.136343010 & -0.049918004\end{array}$




\section{Coord.8}

$\begin{array}{llll}\mathrm{C} & -3.383566241 & -1.810190128 & 0.021621002 \\ \mathrm{C} & -2.998045216 & -0.497636036 & 0.107124007 \\ \mathrm{C} & -1.636578117 & -0.177622011 & 0.057829003 \\ \mathrm{C} & -0.657004046 & -1.203016085 & -0.055653005 \\ \mathrm{C} & -1.090839081 & -2.512611182 & -0.150480012 \\ \mathrm{C} & -2.436263177 & -2.823659204 & -0.120358010 \\ \mathrm{C} & 1.062905078 & 0.314876021 & -0.005697001 \\ \mathrm{C} & 0.169610013 & 1.417773104 & 0.074813005 \\ \mathrm{C} & 0.637538044 & 2.791388202 & 0.120401011 \\ \mathrm{C} & 2.945640211 & 1.874410137 & 0.041817004 \\ \mathrm{H} & -3.721561270 & 0.286688019 & 0.214640018 \\ \mathrm{H} & -0.348475024 & -3.281612238 & -0.249818017 \\ \mathrm{H} & -2.743639195 & -3.847461274 & -0.206635016 \\ \mathrm{H} & 2.402319171 & 3.807881271 & 0.116313006 \\ \mathrm{H} & -4.429190317 & -2.056444148 & 0.059202006 \\ \mathrm{~N} & 0.706561051 & -0.927202066 & -0.074967006 \\ \mathrm{~N} & -1.147162082 & 1.084799078 & 0.096910008 \\ \mathrm{~N} & 2.415129176 & 0.620103045 & 0.000436000 \\ \mathrm{~N} & 2.016304143 & 2.890233210 & 0.093206005 \\ \mathrm{O} & 4.118865294 & 2.088326151 & 0.036397002 \\ \mathrm{O} & -0.068531004 & 3.752106270 & 0.170827010\end{array}$




\section{Coord.9}

$\begin{array}{llll}\text { C } & -3.383526246 & -1.811676132 & 0.022726004 \\ \text { C } & -2.997783215 & -0.498069035 & 0.107498008 \\ \text { C } & -1.637525117 & -0.176674011 & 0.058248006 \\ \text { C } & -0.657756049 & -1.204104089 & -0.055230002 \\ \text { C } & -1.089803079 & -2.511206179 & -0.149298010 \\ \text { C } & -2.437385175 & -2.823866202 & -0.119055007 \\ \text { C } & 1.062619074 & 0.314770021 & -0.005753998 \\ \text { C } & 0.168293013 & 1.416888102 & 0.074450005 \\ \text { C } & 0.637304047 & 2.791130202 & 0.119721008 \\ \text { C } & 2.945488210 & 1.874197133 & 0.041503001 \\ \mathrm{H} & -3.721824266 & 0.285915018 & 0.214291015 \\ \mathrm{H} & -0.347595024 & -3.280449238 & -0.247973020 \\ \mathrm{H} & -2.743456195 & -3.848014275 & -0.205136016 \\ \mathrm{H} & 2.401864174 & 3.807701272 & 0.115401006 \\ \mathrm{H} & -4.429222316 & -2.057676146 & 0.060716004 \\ \mathrm{~N} & 0.706734049 & -0.927488065 & -0.074579003 \\ \mathrm{~N} & -1.150489083 & 1.086501076 & 0.096424006 \\ \mathrm{~N} & 2.415046175 & 0.620008047 & 0.000375001 \\ \mathrm{~N} & 2.015960146 & 2.889934209 & 0.092610009\end{array}$



0
4.118596298
2.088401151
0.036139001
0
$-0.068571005$
3.751646272
0.169680013
$\mathrm{H}$
3.062042219
$-0.136732009$
$-0.049120005$

\section{Coord.10}

$\begin{array}{llll}\text { C } & -3.385218243 & -1.812135130 & 0.023607999 \\ \text { C } & -2.999254217 & -0.497479034 & 0.107677008 \\ \text { C } & -1.640204119 & -0.174700010 & 0.058480002 \\ \text { C } & -0.660241050 & -1.204164087 & -0.055015003 \\ \text { C } & -1.090499079 & -2.508770181 & -0.148353011 \\ \text { C } & -2.440238176 & -2.823042204 & -0.117997007 \\ \text { C } & 1.060600078 & 0.315692023 & -0.005987000 \\ \text { C } & 0.165241009 & 1.417028102 & 0.073935005 \\ \text { C } & 0.635335047 & 2.791896202 & 0.118919008 \\ \mathrm{C} & 2.943602212 & 1.875011135 & 0.041048004 \\ \mathrm{H} & -3.723819269 & 0.286164022 & 0.213763018 \\ \mathrm{H} & -0.348448026 & -3.278253237 & -0.246382017 \\ \mathrm{H} & -2.745005197 & -3.847535274 & -0.203904017 \\ \mathrm{H} & 2.399675174 & 3.808545272 & 0.114389007 \\ \mathrm{H} & -4.430986318 & -2.057882150 & 0.062000005 \\ \mathrm{~N} & 0.705173051 & -0.926745068 & -0.074393008 \\ \mathrm{~N} & -1.155550081 & 1.089227080 & 0.095778008\end{array}$




$\begin{array}{llll}\mathrm{N} & 2.413228176 & 0.620941045 & 0.000146000 \\ \mathrm{~N} & 2.013880146 & 2.890660208 & 0.091894005 \\ \mathrm{O} & 4.116593298 & 2.089502152 & 0.035747003 \\ \mathrm{O} & -0.070345003 & 3.752209269 & 0.168430012 \\ \mathrm{H} & 3.060150220 & -0.135898009 & -0.048905006\end{array}$

